Cooperative Institute for Research in Environmental Sciences

University of Colorado at Boulder.

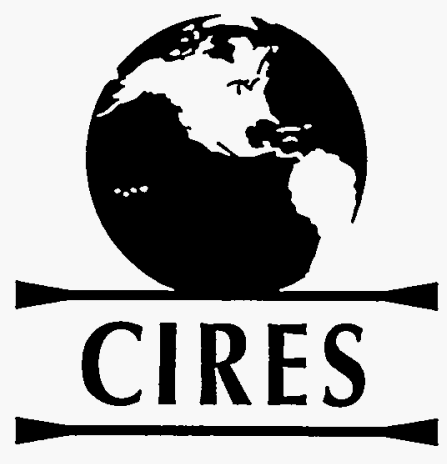

\title{
Thermohaline Circulations and Global Climate Change
}

\section{Final Report}

USDOE Grant No. DE-FG03-93ER61646 Howard P. Hanson, Principal Investigator

October, 1996 


\section{DISCLAIMER}

Portions of this document may be illegible in electronic image products. Images are produced from the best available original document. 


\section{Thermohaline Circulations and Global Climate Change Final Report on USDOE Grant No. DE-FG03-93ER61646}

Howard P. Hanson, Principal Investigator

Cooperative Institute for Research in Environmental Sciences

University of Colorado at Boulder, 80309-0216

\section{Contents}

Summary

1. Introduction $\quad 2$

2. The MICOM

3. Atmospheric Forcing of Ocean Circulation Models $\quad 7$

a. Natural boundary conditions $\quad 7$

b. Scales of forcing $\quad 27$

4. MICOM Results: Oceanic Responses to Global Warming 35

a. Sector model experiments $\quad 36$

b. Atlantic-basin model experiments 44

$\begin{array}{ll}\text { 5. Conclusion } & 55\end{array}$

6. Bibliography 


\title{
Thermohaline Circulations and Global Climate Change Final Report on USDOE Grant No. DE-FG03-93ER61646
}

\author{
Howard P. Hanson, Principal Investigator \\ Cooperative Institute for Research in Environmental Sciences \\ University of Colorado at Boulder, 80309-0216
}

\section{Summary}

This report discusses results from the project entitled Thermohaline Circulations and Global Climate Change, which was funded under two separate DOE awards over the period 15 July 1990 - 14 July 1996 (this period includes a no-cost extension year). Formally, this Final Report is concerned with the latter of the two awards (referenced above); however, the work itself was continuous over the entire period of funding, so this document discusses results in a seamless manner. The first award (DOE Grant No. DE-FG02-90ER61019, for the period $7 / 15 / 90-6 / 14 / 94$, including a no-cost extension year)) was closed out with a separate Final Report in the fall of 1994.

Results are discussed in three sections related to development of the Miami Isopycnic Coordinate Ocean Model (MICOM), surface forcing of the ocean by the atmosphere, and experiments with the MICOM related to the problem of the ocean's response to global climate change. It will require the use of a global, coupled ocean-atmosphere climate model to quantify the feedbacks between ocean and atmosphere associated with climate changes. The results presented here do provide guidance for such studies in the future. 
USDOE Grant No. DE-FG03-93ER61646

Final Report

Page 2

\section{Introduction}

The project entitled "Thermohaline Circulations and Global Climate Change" was originally funded under the USDOE Carbon Dioxide Research Program via Grant No. DE-FG02-90ER61019 (for the period 7/91-7/93); the research was continued under the present award for the period 7/93-7/76 (including the no-cost extension year). The main focus of the research involved addressing the question of whether changes in atmospheric forcing of the ocean circulation (particularly the thermohaline circulation) would be amplified or damped by the oceanic response. That is, the research investigated the hypothesis that changes in surface thermal and hydrological forcing of the North Atlantic, changes that might be expected to accompany $\mathrm{CO}_{2}$-induced global warming, could result in ocean-atmosphere interactions' exerting a positive feedback on the climate system. Because the North Atlantic is the source of much of the global ocean's reservoir of deep water, and because this deep water could sequester large amounts of anthropogenically produced $\mathrm{CO}_{2}$, changes in the rate of deep-water production are important to future climates. Since deep-water production is controlled, in part, by the annual cycle of the atmospheric forcing of the North Atlantic, and since this forcing depends strongly on both hydrological and thermal processes as well as the wind stress, there is the potential for feedback between the relatively short-term response of the atmosphere to changing radiative forcing and the longer-term processes in the oceans. The potential feedback is illustrated in Fig. 1.1.

Feedback processes in the climate system are what makes it so difficult to understand and challenging to study. The potential for global warming induced by the build-up of radiatively active trace gases in the atmosphere was not appreciated until the feedback associated with the atmosphere's maintaining a fixed relative humidity profile was included in model studies. Other feedbacks, involving snow/ice cover and the surface albedo, clouds (both as sunlight reflectors and infrared absorbers), the biosphere, the atmospheric circulation, and atmospheric aerosols and 
USDOE Grant No. DE-FG03-93ER61646

Final Report

Page 3

their relationships to surface properties as well as human activity, have provided scientists with a host of challenges in their attempts to understand fully the climate system. Processes that lead to positive feedback - amplification of changes-are those of most concern, because they tend to destabilize the system; examples include the fixed relative humidity distribution and the snow/ice-albedo linkage. On the other hand, it is clear that negative feedback dominates (after all, the Earth is not ice-covered, nor is it similar to Venus); this is due to the strength of the fundamantal physics of radiation to space (as approximated by the fourth-power StefanBoltzmann radiation law). However, predicting global change quantitatively depends critically on understanding the effects of all feedbacks on the overall system, and, because the oceanatmosphere interaction processes of interest here have the potential for positive feedback, they are of relevance to the global climate change problem.

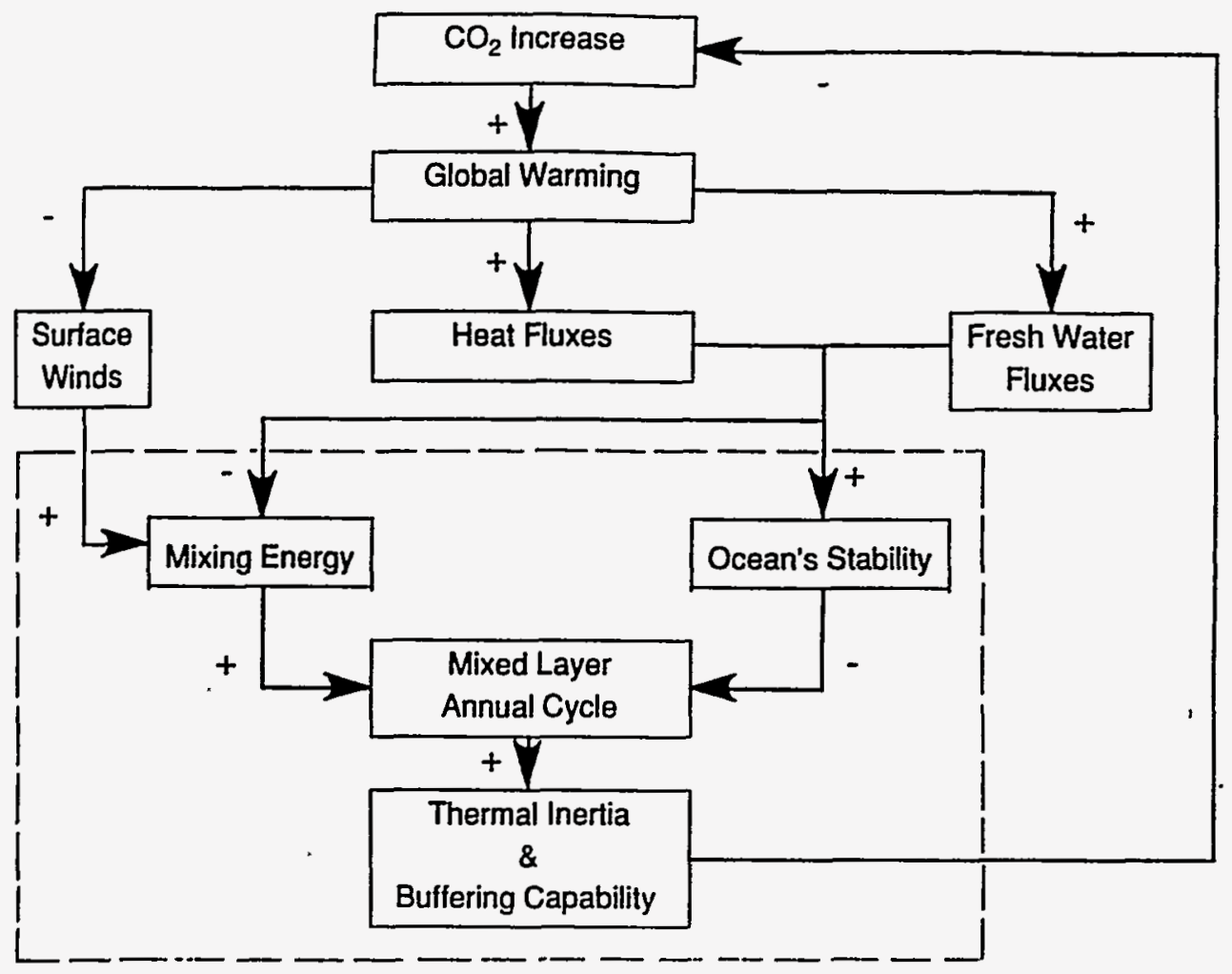

Figure 1.1 Schematic diagram of ocean-atmosphere feedback process in this project. The sense of each component of the overall feedback loop is indicated by the "+" or "." signs, which imply amplification or damping, respectively. 
USDOE Grant No. DE-FG03-93ER61646

Final Report

Page 4

Over the six years of work funded by these awards, several aspects of the overall problem were addressed, and much of the effort involved improving the modeling tools available to address the problem as well as other aspects of the bigger issue of global change. In particular, significant effort was devoted to the development of the Miami Isopycnic Coordinate Ocean Model (MICOM), and the payoff for this investment will be seen in the model's use in a variety of other projects (both DOE-supported and otherwise).

From a theoretical perspective, complete investigation of the feedback process depicted in Fig. 1 requires an interactive ocean-atmosphere global model, and the investment in time and effort in this approach was deemed premature for this project. Accordingly, the oceanic part of the feedback process (that part confined to the dashed box in Fig. 1.1) was investigated alone, using the MICOM in various versions. This effort was assigned to Ms. Fiona Horsfall, a doctoral student at the Rosenstiel School for Marine and Atmospheric Sciences at the University of Miami, under a subcontract supervised by Prof. Rainer Bleck, a co-PI on the project. Ms. Horsfall's results, discussed at length below, show that this approach was justified, because it appears that the net effect of the potential feedback is small. While future models of the climate system will implicitly include these processes (provided, of course that they employ a fully interactive global climate model including both atmosphere and ocean), it is likely that other processes will be of primary interest.

In the following sections, development of the MICOM over the project period is reviewed briefly, certain aspects of the appropriate forcing of ocean models for climate studies are discussed, and Ms. Horsfall's results concerning the nature of the ocean's response to varying atmospheric forcing is summarized. A bibliography follows. 
USDOE Grant No. DE-FG03-93ER61646

Final Report

Páge 5

\section{The MICOM}

The Miami Isopycnic Coordinate Ocean Model (MICOM) has grown out of an effort begun in the 1980s to exploit the benefits of isopycnic coordinate modeling in ocean circulation studies. Early work (e.g., Bleck and Boudra, 1981, 1986) concentrated on purely wind-driven circulation modeling. As with isentropic models (the atmospheric counterpart of isopycnic coordinate ocean models), including thermodyanamics is "unnatural" in the sense that these processes act as sources or sinks of independent variables. However, modeling the ocean has the advantage that most of the thermodynamics occurs near the surface, rather than in the deep interior of the fluid as does, for example, water phase changes associated with deep convection in the atmosphere.

This characteristic of the oceans was used in the development of a hybrid model using isopycnic coordinates in the deep ocean with a thermodynamically active mixed layer at the surface (Kraus et al., 1988; Bleck et al., 1989). The technical aspects of matching the bottom of the mixed layer with the upper-most isopycnic layer involved carrying in the model computation a variable number of isopycnic layers containing zero mass when the mixed layer density exceeds their coordinate densities. Model detrainment, associated with warming of the mixed layer above the coordinate values, would then add mass to the "deflated" layers in a way that preserves energy and mass. The first versions of the hybrid model used the original KrausTurner (1967) mixed-layer model (e.g., Niiler and Kraus, 1977). More recent versions of the MICOM include both this model as an option and the more recent diffusion and dissipation model developed by Gaspar (1988). Work during this project showed thatthe Gaspar approach provides more reasonable mixed layer behavior (Haliwell et al., 1994). Additional model improvements over the early versions have included realistic basin topography and geography, a free surface upper boundary condition, and diapycnal mixing (the equivalant of vertical diffusion). The latter follows the development of $\mathrm{Hu}$ (1991). 
Page 6

It has been the philosophy of the model developers (primarily R. Bleck and colleagues at the University of Miami) to keep the model freely accessible; at this writing it is available via http://www.rsmas.miami.edu/groups/micom.html on the World-Wide Web. This has led to wide interest in the model as a tool for a variety of oceanographic problems, including flow in channels and high-latitude ocean/ice interaction studies. Interest in the model has become so wide spread that the Miami group began, in 1994, holding annual workshops to discuss the model, its uses, and its future development. In joint work with investigators at DOE laboratories (e.g., Bleck and Dean, 1995), the model has been reprogrammed into a highly parallel mode (Bleck et al., 1995) on global scales (e.g., Bleck et al., in press), and, with the availability of faster computers, is being used for long-term circulation studies with ever higher resolution (e.g., Bleck and New, 1995; Bleck, 1995). The award discussed in this report was in collaboration with the Miami group, and funds provided to Bleck and colleagues via a subcontract were used to support parts of many of these activities.

Results discussed here concentrate on two aspects of the MICOM and its use to investigate the problem outlined in the Introduction. Investigating effects of ocean-atmosphere interaction processes on the oceanic circulation requires that the physics of these processes be formulated properly in the model; this is the subject of the next section. Results pertaining to the sensitivity of the model to changes in surface forcing are then discussed in Section 4 . 
USDOE Grant No. DE-FG03-93ER61646

Final Report

Page 7

\section{Atmospheric Forcing of Ocean Circulation Models}

Whether the atmosphere is viewed as an upper boundary condition or as a source function for ocean modeling, it is necessary to formulate properly the coupling between the media, if realistic simulations of the oceanic circulation are to be obtained. Because this project was concerned with the effects of varying atmospheric forcing on an ocean circulation model, considerable effort was invested in the proper formulation of that forcing. There are two aspects to this, how to simulate the processes of the forcing, and what scales need to be included. Various aspects of the results reported here were discussed in Hanson (1994, 1995a,b,c) Hanson et al. (1996), and Hanson and Bleck (1996).

\section{a. Natural boundary conditions}

Ocean general circulation models (OGCMs) now routinely calculate both the wind-driven circulation and the thermohaline circulation by solving the momentum equations and the conservation equations for heat and salt; the system is closed by the continuity equation and an equation of state. Consequently, the conditions needed at the ocean surface include both wind forcing as well as thermodynamic forcing. Specification of the wind stress at the surface, given an appropriate atmospheric dataset, is relatively straight-forward; specification of the thermodynamic boundary condition is less so because in nature the sea-surface temperature (SST) plays a strong role in determining the surface heat fluxes.

One approach to specifying surface boundary forcing for the heat and salt budgets follows the specification of the wind stresses in the momentum budget and is simply uses the thermodynamic fluxes of fresh water (both precipitation and evaporation) and heat (the sum of net radiation plus sensible and latent heat loss). This flux forcing, however, eliminates an important local degree of freedom for the calculation of salinity and SST. The other extreme is 
USDOE Grant No. DE-FG03-93ER61646

Final Report

Page 8

to specify a surface salinity and temperature, usually derived from climatology, toward which the salinity and SST in the model are forced to relax (e.g., as used in Semtner and Chervin, 1992). Such pure relaxation forcing, even if it uses modified surface temperatures and salinities as suggested, for example, by Haney (1971), eliminates forcing that is not associated with localized relaxation processes, such as solar heating and precipitation. In addition, it also requires salinity and SST climatologies, the use of which bias model behavior. A more realistic, and natural, approach would use a combination of these, in the form of mixed boundary conditions.

Even though mixed boundary conditions are likely to be more realistic than either extreme approach, the real ocean does not relax toward some climatological SST and salinity distribution. Rather, it relaxes toward conditions in the atmosphere. This is easily shown by considering the thermodynamic equation integrated from some depth $h$ at which vertical turbulent transport disappears to the surface:

$$
\int_{-h}^{0} \frac{D T_{S}}{D t} d z=\frac{-1}{\rho_{W} C_{W}}\left[F_{R a d}+H+L E\right] \equiv-Q_{N e t},
$$

where $T_{S}$ is the SST, $D() / D t$ represents the total derivative (in which, for convenience, horizontal diffusion is included), $\rho_{W}$ and $C_{W}$ are the density and specific heat of seawater, and the surface heating is the (negative) sum of the (upward) radiative, sensible, and latent heat fluxes. Each of these fluxes depends on the SST; further, in the most commonly used parameterizations for these fluxes this dependence is positive. That is, including the negative sign in (1) implies functionality of the form $D T_{S} D t \propto-T_{S}$, which implies negative feedback.

Using flux boundary conditions, that is, simply specifying $Q_{\text {nel, }}$ removes this negative 
USDOE Grant No. DE-FG03-93ER61646

Final Report

Page 9

feedback—and its associated stabilizing effect—from the system. The other extreme approach, setting

$$
Q_{N e t}=\gamma\left(T_{S}-T_{*}\right)
$$

(with $T$ * the specified climatological SST and $\gamma$ a relaxation time constant, typically about a week), provides strong negative feedback, of course, and forces the SST to relax toward $T$. A mixed boundary condition approach combines these two extremes, specifying part of the forcing through a flux and retaining the negative feedback of the relaxation toward the specified temperature. However, the actual dependence involves the air temperature, through the bulk formulae; further, it is also dependent on the saturation vapor pressure relationship to temperature and, most likely, on the functional form used to specify the upward infrared (IR) heat loss from the ocean. These issues are discussed in more detail in the following section. Subsequently, the different forms of the surface forcing are compared.

\section{i. Surface Fluxes}

In order to provide as much realism as possible while retaining computational simplicity, it is desirable to formulate the sensible heat flux as

$$
H=\rho_{A i r} C_{P} C_{T} U_{10}\left(T_{S}-T_{A i r}\right),
$$

where $C_{P}, \rho_{A i r}$ and $T_{A i r}$ are the specific heat of air at constant pressure and the density and the temperature of the air near the surface; $U_{10}$ is the near-surface wind speed; and $\zeta$ is a heat transfer coefficient. Comparing (2) and (3) shows how the sensible heat loss at the surface tends to make the SST relax toward the air temperature. Strictly speaking, all of the coefficients leading the temperature difference in (3) except the wind speed have some dependence on the 
USDOE Grant No. DE-FG03-93ER61646

Final Report

Page 10

local air temperature; the transfer coefficient also depends on the SST via the stability (e.g., Smith, 1987).

In developing a surface-forcing climatology from databases such as the Comprehensive Ocean Atmosphere Data Set (COADS; Woodruff et al., 1987), there is an additional consideration: space-time averages of wind, air temperature, and SST, when used in (3), compute fluxes that alias wind and temperature-difference correlations on smaller space and time scales. However, COADS, in which averages are monthly on $2^{\circ}$ latitude-longitude squares, includes these correlations by providing averages of the products of individual observations. Defining the averaged quantity relevant to the sensible heat flux as $\langle U \Delta T\rangle$ (this could be called a pseudoflux, by analogy to the pseudo-stress defined by Trenberth et al., 1989), (3) becomes

$$
H=\rho_{A i r} C_{P} C_{T}<U \Delta T>
$$

Used with observations of the air temperature, humidity, wind speed, and stability to calculate the coefficients as carefully as possible, (4) provides a good estimate of the climatological sensible heat flux for a given month at any location. For long-term integrations of an OGCM, it is clearly preferable to avoid all these extra calculations, and it is further desirable, as discussed above, to include the model temperature in the calculation. This is most easily done by setting the values of the coefficients in (3) to constants (denoted by ${ }_{0}$ subscripts) and equating (3) and (4) to find an effective air temperature $T_{E f f}$

$$
T_{E f f} \equiv T_{S}-\frac{\rho_{A i r} C_{P} C_{T}<U \Delta T>}{\rho_{0} C_{P o} C_{T o} U_{10}} .
$$

This leads to a form for the sensible heat flux in an OGCM integration that, provided the model 
USDOE Grant No. DE-FG03-93ER61646

Final Report

Page 11

calculates the SST properly, reproduces the climatological sensible heat flux exactly:

$$
H_{\text {Model }}=\rho_{0} C_{P_{o}} C_{T_{0}} U_{10}\left(T_{\text {Model }}-T_{E f f}\right) \text {, }
$$

where $T_{E f f}$ from (5) and $U_{I 0}$ vary with space and time. Note that by embedding the variations
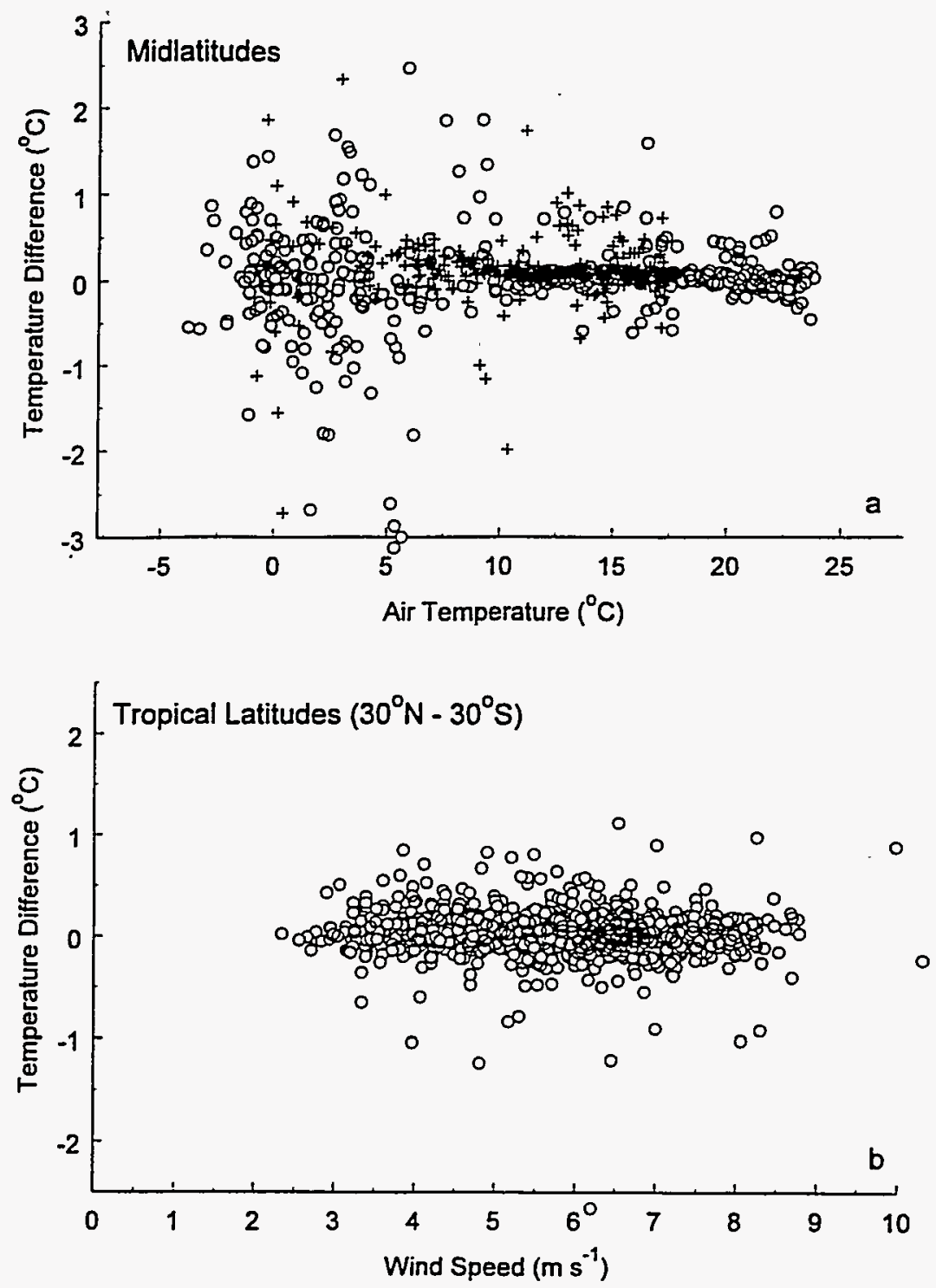

Figure 3.1 The temperature difference $T_{E f f}-T_{A i r}\left({ }^{\circ} \mathrm{C}\right)$ along a four grid-point wide merdional transect through the North and South Atlantic centered on $20^{\circ} \mathrm{W}$. Fig. 3.1a shows the temperature difference vs. $T_{A i r}$ for latitudes poleward of $30^{\circ}$; circles are "summer" months in both hemispheres and crosses are "winter" months. Fig. 3.1b shows the temperature difference vs. wind speed for tropical and subtropical latitudes for all months. 
USDOE Grant No. DE-FG03-93ER61646

Final Report

Page 12

of the air density, its specific heat, and the transfer coefficient in the definition of $T_{E f \text { f }}$, this approach avoids problems associated with inappropriate estimates of the heat flux analogous to those associated with the wind stress discussed by Trenberth et al. (1989).

Figures 3.1 show $T_{E f f}-T_{A i r}$, using data from the COADS long-term (1950-1979) mean forcing set on a four grid-point-wide meridional transect through the North and South Atlantic centered on $20^{\circ} \mathrm{W}$. A useful rule of thumb to relate sensible heat flux errors to temperature biases is that, for a $7.5 \mathrm{~m} \mathrm{~s}^{-1}$ wind speed, a $1^{\circ} \mathrm{C}$ temperature difference translates to about $10 \mathrm{~W}$ $\mathrm{m}^{-2}$. Figure 3.1a shows $T_{E f f}-T_{A i r}$ plotted against $T_{A i r}$ for "winter" and "summer" in crosses and circles, respectively, for latitudes poleward of $30^{\circ}$ in both hemispheres. The months used for these "seasons", January-March and July-September, were chosen to account for the SST time lag behind the solar seasons. The greatest RMS temperature differences, which, given sufficient wind speed, could be associated with flux errors of more than $30 \mathrm{~W} \mathrm{~m}^{-2}$, tend to correlate with lower air temperatures, reflecting the greater variability at higher latitudes on small time and space scales.

Figure 3.1b shows $T_{E f f}-T_{A i r}$ plotted against wind speed for all months for the tropical and subtropical regions. Climatologically, winds in the tropics tend to be lower than in the higher latitudes, and, given the form of (5), it might be the case that lower wind speeds cause large excursions in the $T_{E f f}$ calculation. Figure $1 \mathrm{~b}$ shows that this is not so. Note also in Figs. $1 \mathrm{a}$ and $1 \mathrm{~b}$ that there is no apparent trend of the temperature difference associated with either wind speed or air temperature.

The latent heat flux, by analogy to (6) in the derivation above, becomes

$$
L E_{\text {Model }}=\rho_{0} L_{0} C_{T o} U_{10}\left(q *\left(T_{\text {Model }}, P_{S}\right)-q_{E f f}\right) \text {, }
$$


USDOE Grant No. DE-FG03-93ER61646

Final Report

Page 13
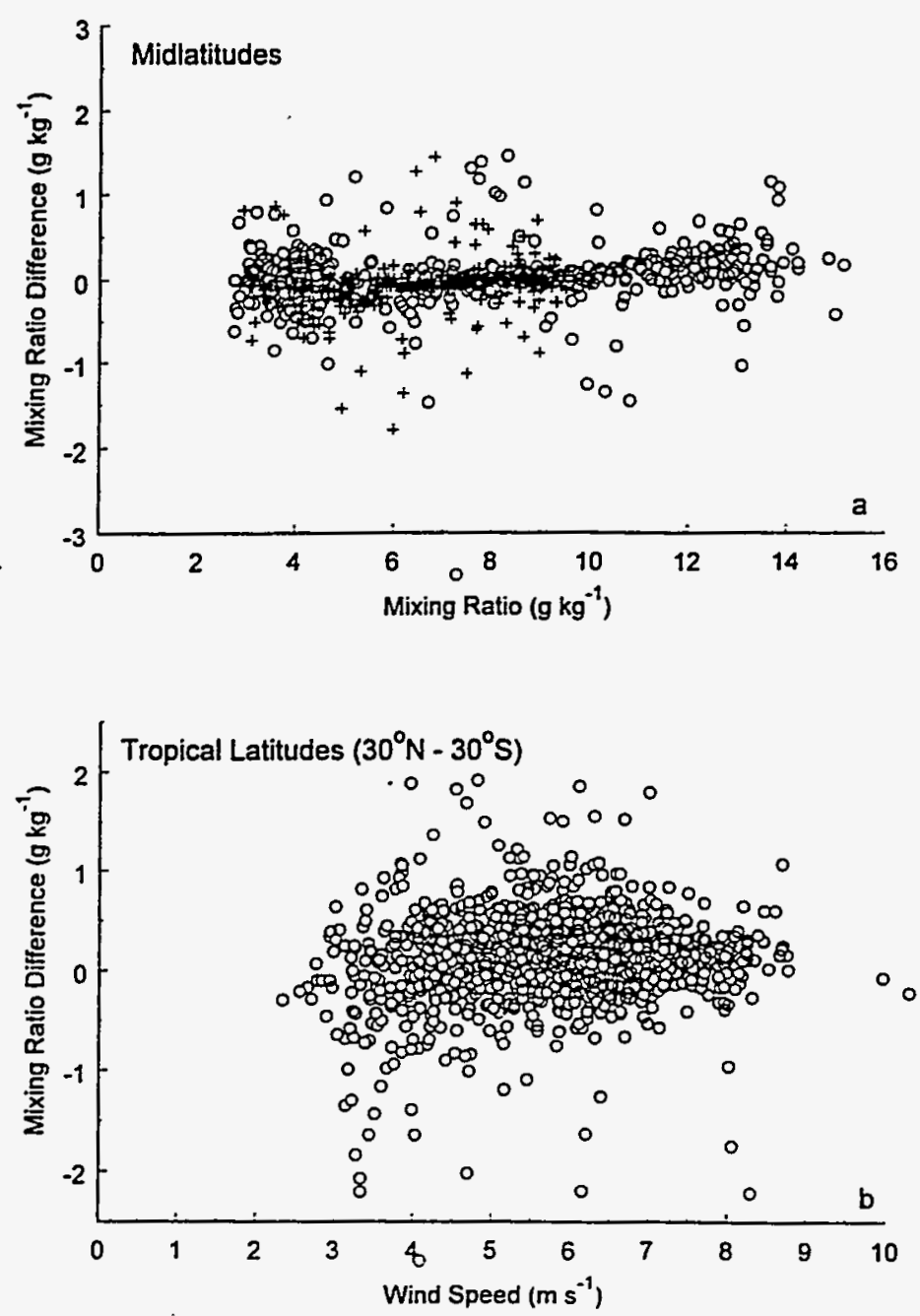

Figure 3.2 As in Fig. 3.1, except for the water vapor mixing ratio difference $q_{E f}-q_{A i r}$ (g $\left.\mathrm{kg}^{-1}\right)$.

with

$$
q_{E f f} \equiv q *\left(T_{S}, P_{S}\right)-\frac{\rho_{\text {Air }} L C_{E}<U \Delta q>}{\rho_{0} L_{0} C_{T o} U_{10}}
$$

Here, $q$ is the water vapor mixing ratio, $q^{*}$ is its saturation value, and $C_{E}$ is the transfer coefficient for water vapor. Figures 3.2, which are the mixing-ratio analogs of Figs. 3.1, show 
USDOE Grant No. DE-FG03-93ER61646

Final Report

Page 14

that in the midlatitudes latent-heat flux calculations can be biased by as much as about $50 \mathrm{~W} \mathrm{~m}^{-2}$ by neglecting subgrid, sub-monthly correlations of water vapor and wind speed (the analogous rule of thumb being that a mixing-ratio error of $1 \mathrm{~g} \mathrm{~kg}^{-1}$ gives a flux error of about $25 \mathrm{~W} \mathrm{~m}^{-2}$ for a $7.5 \mathrm{~m} \mathrm{~s}^{-1}$ wind speed). Although there is a slightly greater tendency for larger mixing-ratio differences at low wind speed in the tropics than in the case of temperature (Fig.3. 2b), this is not significant.

In (7) and (8) $q^{*}(T, P)$ is the saturation mixing ratio, a highly nonlinear function. Because it enters the model integration at each grid point at each time step via (7), modelers seeking computational efficiency are motivated to find methods to avoid calculating $q^{*}$. For example, in Bleck et al. (1989) we used an atmospheric pseudo-temperature calculated from

$$
\hat{T}_{A i r} \equiv T_{S}-\frac{\rho_{A i r}\left(C_{P} C_{T}<U \Delta T>+L C_{E}<U \Delta q>\right)}{\rho_{0} C_{P o} C_{T o} U_{10}}
$$

to combine the sensible and latent heat fluxes into the simple expression

$$
(H+L E)_{\text {Model }}=\rho_{0} C_{P o} C_{T o} U_{10}\left(T_{\text {Model }}-\hat{T}_{A i r}\right) .
$$

Oberhuber (1993) developed an analogous method by linearizing the net heat flux about an optimized pseudo-temperature. However, while this expression and Oberhuber's method are computationally efficient, they omit the dependence of the saturation mixing ratio on the model SST in (7) and therefore strongly underestimate the feedback associated with model SST errors. This is shown in Fig. 3.3, which compares the results of using (4) + (7) on the abscissa with (10) on the ordinate for the observations (circles) and for SSTs lowered $(-)$ and raised $(+)$ by $2^{\circ} \mathrm{C}$. The circles align, of course, because of the way in which (4) and (7) can be combined to yield (10). However, when SST errors are introduced, as happens in model calculations, the flux errors can 
USDOE Grant No. DE-FG03-93ER61646

Final Report

Page 15

be quite large, as much as $100 \mathrm{~W} \mathrm{~m}^{-2}$ in some cases. ' That the upward flux calculated from (9) is too large for low SSTs and too small for high SSTs (positive fluxes implying oceanic cooling here) shows how the feedback is underestimated in each case.

The computational expense of the $q^{*}$ calculation should therefore be considered to be an investment in greater accuracy for the $L E$ calculation, which affects both the heat and salinity budgets. In that regard, using (9) to calculate evaporation for use in a salinity budget requires specifying yet another parameter from climatology, such as a Bowen ratio. Consequently, the use of (4) and (7) is

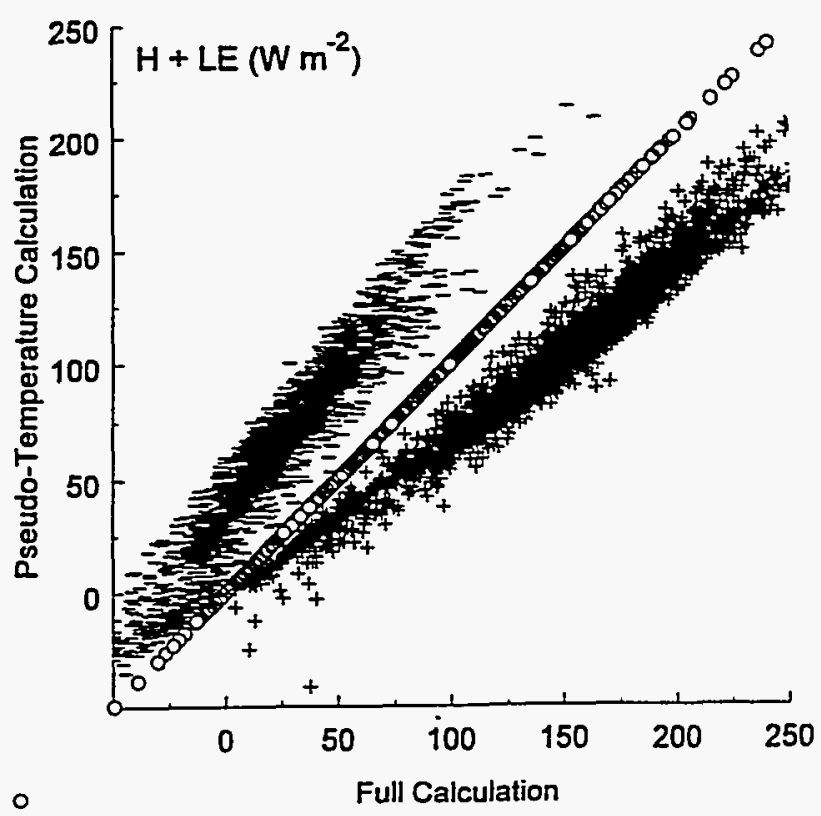

Figure 3.3 Comparison of the turbulent sensible and latent heat fluxes calculated from the pseudo-temperature formulation (Eq. 10) with the sum of the separate calculations (i.e., Eq. $6+$ Eq. 7). Data is taken from the transect used for Figs. $1 \& 2$. Circles show the calculation using the observed SST; pluses and minuses show same calculation with SST increased and decreased by $2^{\circ} \mathrm{C}$, respectively.

recommended. If avoiding nonlinear functions in the computation is desired, sufficient accuracy can be provided by the use of a polynomial approximation for saturation vapor pressure, such as that developed by Lowe (1977), rather than the traditional Goff-Gratch (1946) formulation.

The solar flux and the IR flux emanating from the atmosphere heat the ocean; upward IR emission from the ocean surface cools it. Although the ocean-atmosphere system interacts to determine all of these radiation streams, the local SST has the strongest effect on the upward IR emission from the surface. The approach taken here is therefore to specify the solar and downward IR components and to calculate of the upward IR flux. This is done indirectly, based 
USDOE Grant No. DE-FG03-93ER61646

Final Report

Page 16

on previous work by Oberhuber (1988), who calculated the radiative fluxes from COADS data using empirical formulae developed several decades ago. Oberhuber used, for the IR flux calculation, a formula developed by Berliand (1952) and refined by Budyko (1974)

$$
\begin{aligned}
F_{I R}=\epsilon \sigma T_{A i r}^{4} & (0.39-0.05 \sqrt{e / 100})\left(1-\chi n^{2}\right) \\
+ & 4 \in \sigma T_{A i r}^{3}\left(T_{S}-T_{A i r}\right),
\end{aligned}
$$

where $\epsilon$ is the ocean-surface IR emissivity $(=0.97), \sigma$ is the Stefan-Boltzmann constant, $e$ is the atmospheric water vapor pressure, $n$ is the cloud fraction, and $\chi$ is an empirical factor introduced by Budyko that varies with latitude. The net radiative flux is then the sum of the solar and IR components, $F_{R a d}=F_{I R}+F_{S o l}$. In order to include the model SST dependence in this component of the net surface heating $Q_{N e t}$ by using quantities defined previously, the last term in (11) can be identified with the net effect of the upward IR emission and we can write

$$
F_{\text {Down }} \equiv F_{\text {Rad (Oberhuber) }}-4 \in \sigma T_{E f f}^{3}\left(T_{S}-T_{E f f}\right) \text {. }
$$

This allows calculation of the net radiative flux for modeling purposes as

$$
F_{\text {Rad (Model) }}=F_{D o w n}+4 \in \sigma T_{E f f}^{3}\left(T_{\text {Model }}-T_{E f f}\right) .
$$

Using (6), (7), and (13) developed from an appropriate climatology in an OGCM heat budget, as well as (7) and a precipitation climatology in the salinity budget, provides a climatologically consistent, physically realistic set of natural thermodynamic boundary conditions. The salinity budget, interestingly, becomes strongly coupled to the heat budget via (7); in the case of either pure flux or pure relaxation forcing, this coupling is missing from the model. Other differences among the three types of surface forcing, discussed below, also argue for the use of these mixed boundary conditions. 
USDOE Grant No. DE-FG03-93ER61646

Final Report

Page 17

\section{ii. Forcing Comparison}

The errors introduced by neglecting small-scale correlations of wind and temperature (and wind and water vapor) and by attempting to avoid the computation of the SST saturation humidity can be significant, as shown above. Additional errors are introduced by the very form of the surface forcing. This is shown here using a very simple calculation of the annual cycle of the SST.

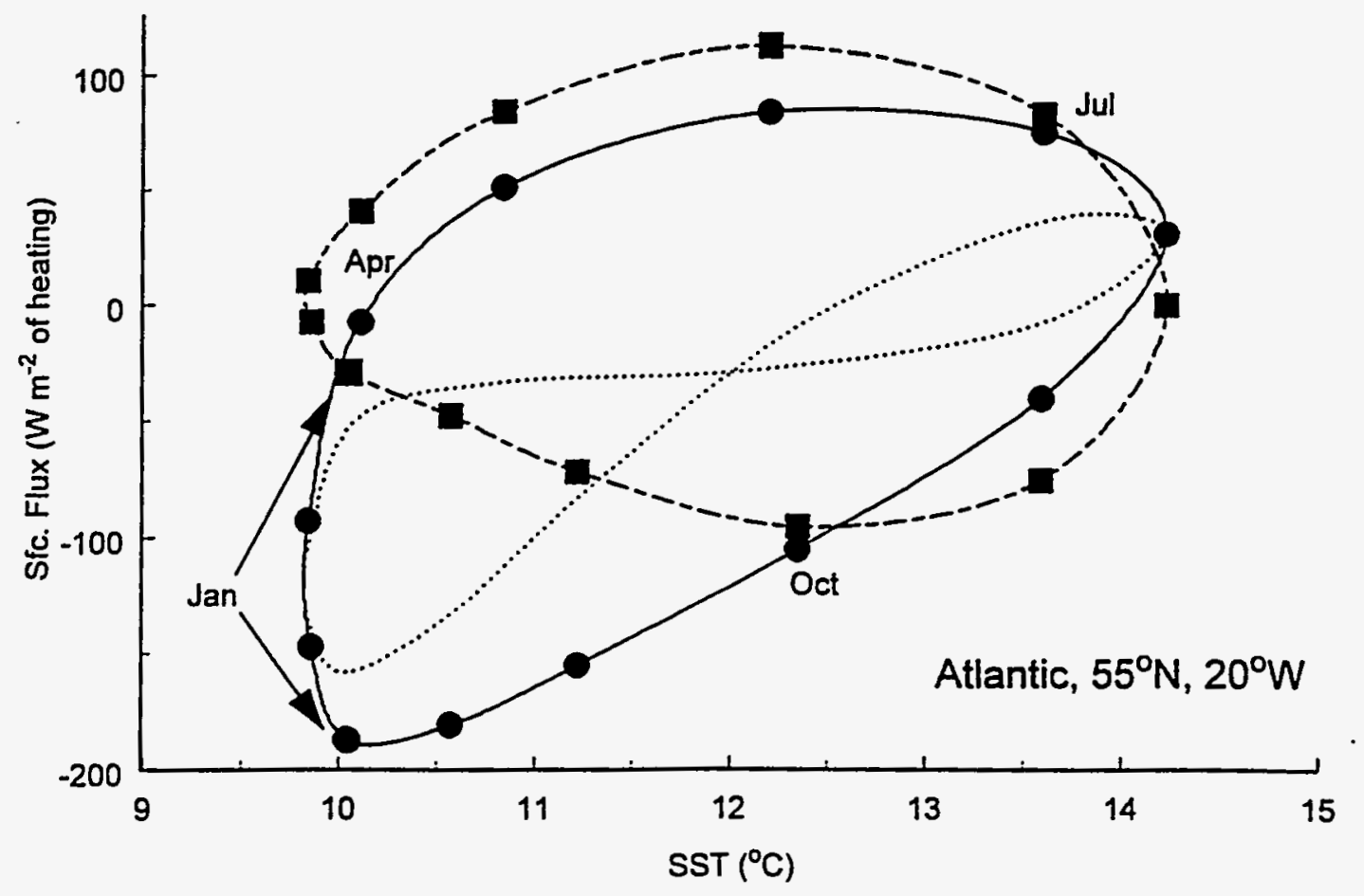

Figure 3.4 Phase diagram comparison for the zone $50^{\circ}-60^{\circ} \mathrm{N}$ along the $20^{\circ} \mathrm{W} 4$ grid-point COADS transect; the ordinate has been transformed to flux units. The solid curve with circles shows the mean annual cycle of the net surface heating vs. SST; the dashed curve with squares shows the actual phase diagram of $\partial \mathrm{T} / \partial \mathrm{t}$ (in flux units) vs. SST; and dotted curve is the difference. The solid curve can be constructed purely from observations; the other two depend on specification of the mixed-layer depth. 
USDOE Grant No. DE-FG03-93ER61646

Final Report

Page 18

In order to illustrate clearly the differences in OGCM behavior that would be associated with the different forms of surface forcing, it is convenient to introduce a graphical construct based on the engineering phase diagram, in which a function (in this case, SST as a function of time) is plotted against its derivative. In the oceanographic literature, this approach was used by Gill and Turner (1976) and Bretherton (1982) in a slightly different form (they used the vertical integral of temperature vs. temperature) to discuss hysteresis associated with the behavior of the annual cycle of the oceanic mixed layer. In this paper, the net surface flux (expressed as heating) is plotted against the SST. Because the surface flux represents only a component - albeit a significant component - of the time derivative of the SST, the figures shown here can be called partial phase diagrams. In all cases, data from the COADS long-term means, on $2^{\circ}$ latitude-longitude squares, are used with the radiative forcing developed by Oberhuber (1988) modified via (12) and (13).

The solid closed curve in Fig. 3.4 is a partial phase diagram of the surface flux / SST relationship for the $50^{\circ}-60^{\circ} \mathrm{N}$ squares in the meridional transect of the Atlantic ap $20 \mathrm{~W}$ discussed previously (so that there is a total of 20 of the COADS/Oberhuber $2^{\circ}$ latitude-longitude squares in this average). The monthly values shown by the circles (January, April, July, and October are labeled) are joined by a cubic-spline interpolation. The lag of the SST associated with the mixed-layer's thermal inertia behind the seasonal cycle of surface heating is apparent. It is emphasized that it is possible to construct this curve purely from observations of oceansurface quantities (although, in this case, the radiative heating has been calculated from empirical formulae).

The SST thermodynamic budget (1) can be rewritten as

$$
\rho_{W} C_{W} h_{0} \frac{\partial T_{S}}{\partial t}=-\left(F_{S f c}-F_{H}+F_{A d \nu}\right)
$$


USDOE Grant No. DE-FG03-93ER61646

Final Report

Pagé 19

where $h_{0}$ is the depth of the surface mixed layer, $F_{S f c}$ is the term in square brackets in (1), $F_{H}$ is the entrainment flux at the base of the mixed layer, and $F_{A d v}$ is the flux associated with horizontal advection and diffusion. If $h_{0}$ is specified as a constant (50 m is used here) and the SST observations are differentiated numerically, a true phase diagram of $\partial T / \partial t$ vs. $T$ can be constructed (where the constants $\rho_{W} C_{W} h_{0}$ multiplying the former give it flux units); this is shown by the squares and the dashed curve in Fig. 3.4. The difference between these curves (dotted, with symbols omitted, in Fig. 3.4) is then $F_{H}-F_{A d v}$. It is important to emphasize that only the solid curve can be constructed purely from observations; the others depend crucially on the specification of $h_{0}$ (which could be a function of time as well, although this would destroy the phase-diagram nature of the curves). The choice of $h_{0}=50 \mathrm{~m}$ used here is motivated by the approximate match of the shapes of the solid and dashed curves in Fig. 3.4.

The utility of these partial phase diagrams is twofold. First, as illustrated by Fig. 3.5, the annual cycle of the SST and surface heating exhibits, when normalized, a very consistent behavior over large areas of the oceans. The quantities plotted in Fig. 3.5 are all normalized according to the formula $X_{N}=\left(X-X_{A v g}\right) / A_{X}$ with $A_{X}$ the amplitude of the annual cycle. The averages and standard deviations used in this normalization are listed in Table $\mathrm{I}$, and the solid curves in Figs. $3.4 \& 3.5$ are identical. Although the behavior of these partial phase diagrams is not truly universal (there are large deviations in the tropics due to the semi-annual cycle, and, of course, the annual phase is inverted in the Southern Hemisphere), the general shape describes most of the midlatitude oceans. Second, the diagrams depict the aspects of the annual cycle that are most important for climate-related ocean studies- the SST and the net surface flux. The phase of the various months on the diagrams provides an especially useful tool for diagnosing model behavior, as shown next. It is worth emphasizing that the superposition of the normalized plots is due entirely to the normalization, and that the original data show the range of both SST and surface heating expected for the latitude range used (Fig. 3.6). 


\section{Final Report}

Page 20

Normalizations use mean, amplitude of annual cycles of observations
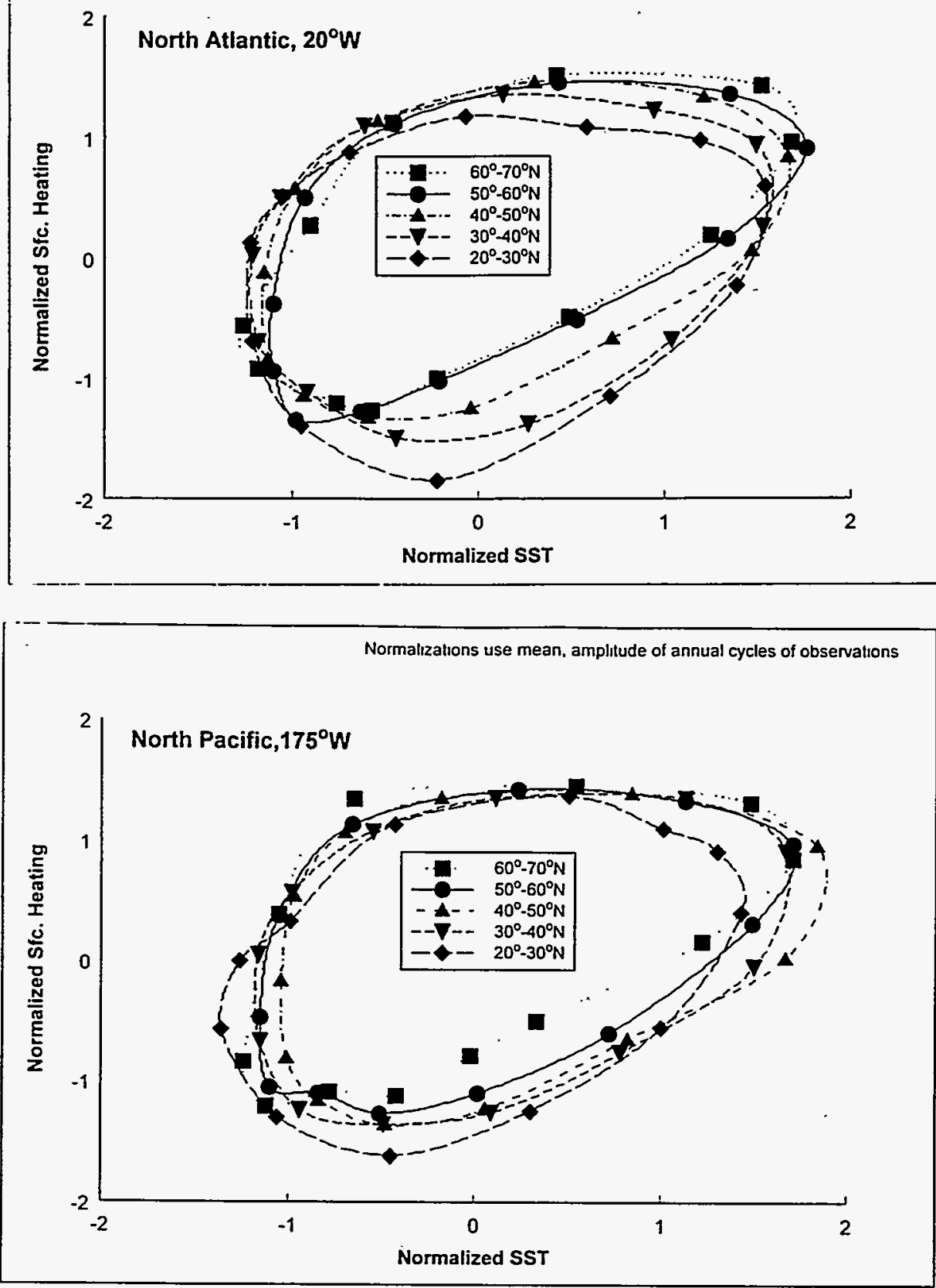

Figure 3.5 Partial phase diagrams (as in the solid curve in Fig. 3.4), using data normalized by averages and annual amplitudes, from the other latitide zones on the $20^{\circ} \mathrm{W}$ transect in the N. Atlantic (top) and along a similar transect along $175^{\circ} \mathrm{W}$ in the N. Pacific (bottom). 
USDOE Grant No. DE-FG03-93ER61646

Final Report

Page 21
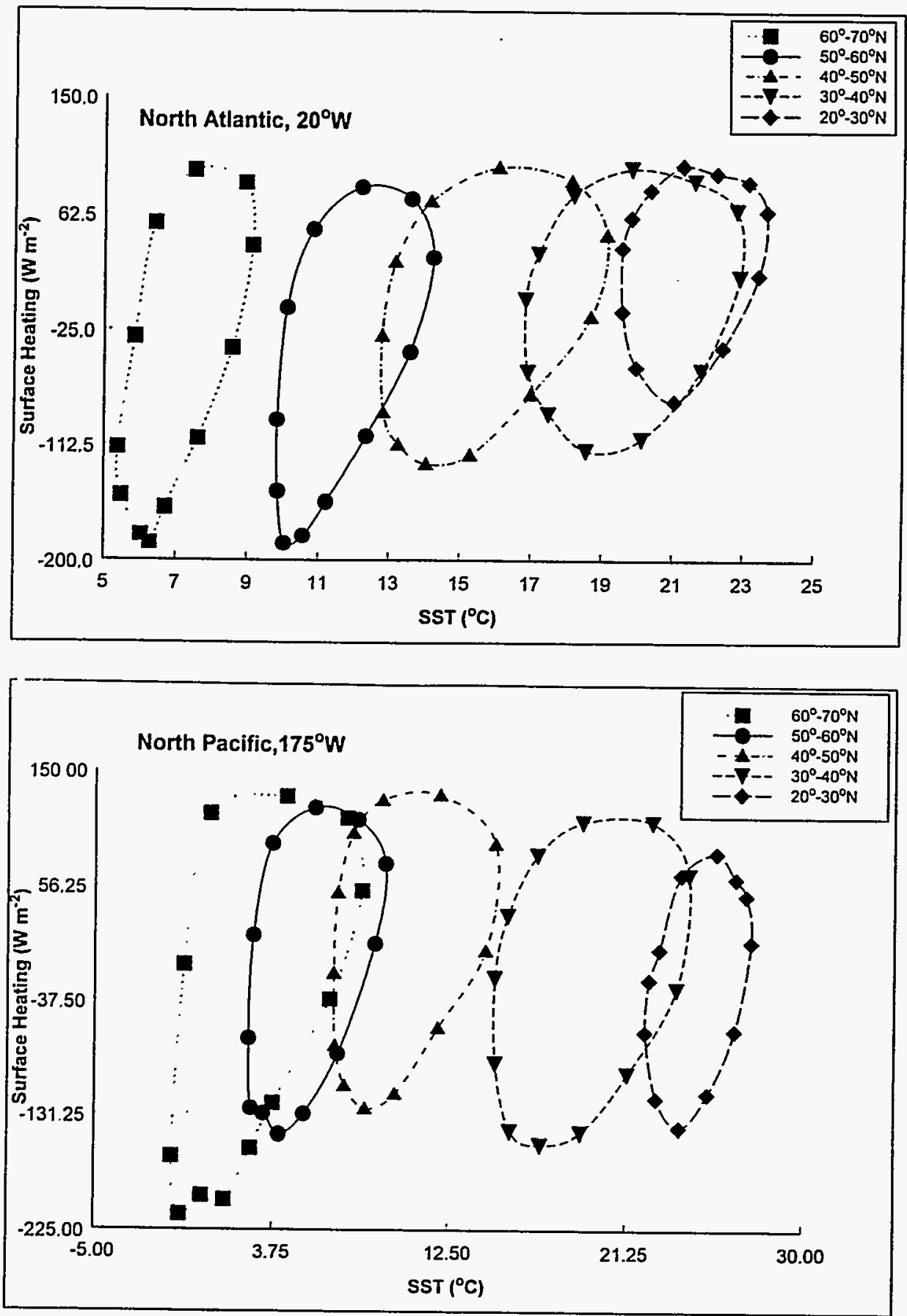

Figure 3.6 Original data used to construct normalized plots of Fig. 3.5 
USDOE Grant No. DE-FG03-93ER61646

Final Report

Page 22

Figures 3.7-3.9 show results of integrating (14) with the $F_{H}+F_{A d v}$ term taken from the dotted curve in Fig. 3.4 and the surface flux term calculated with the three types of forcing discussed in Section 2. In each case, the initial conditions and forcing are taken from the observations in the $50^{\circ}-60^{\circ} \mathrm{N}$ zone of the $20^{\circ} \mathrm{W}$ Atlantic transect; integrations made as well with $\pm 2^{\circ} \mathrm{C}$ perturbations to the initial conditions. The observations from Fig. 3.4 (circles on solid curves) are compared with the unperturbed (squares on dashed curves) and the perturbed runs $(+/$ - symbols on dotted curves). In all cases a fourth-order predictor-corrector method of integration was used with a one-day time step, and $h_{0}$ was held at $50 \mathrm{~m}$.

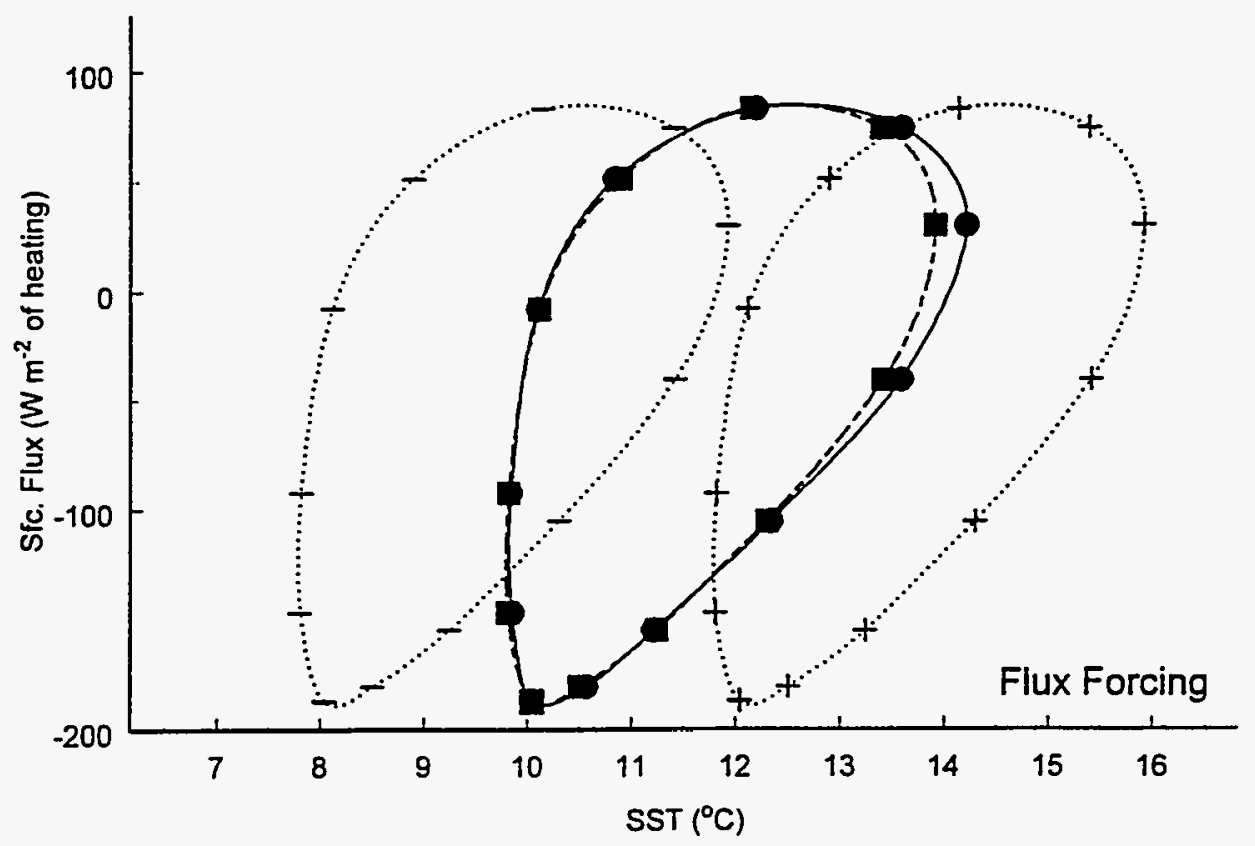

Figure 3.7 Observations from Fig. 3.4 (solid) with the results of integration Eq. 14 using flux forcing for unperturbed initial conditions (dashed) and initial conditions perturbed by $\pm 2^{\circ} \mathrm{C}$ (dotted). 
USDOE Grant No. DE-FG03-93ER61646

Final Report

Page 23

Flux forcing (Fig.3.7), in which the ordinate values of the observations in Fig. 3.4 are used on the right-hand-side of (14), reproduces the observationsof SST rather well when the initial conditions are unperturbed, with errors of about $1 / 3^{\circ} \mathrm{C}$ in the summer and small phase errors. These errors can be attributed to a combination of the constant mixed-layer depth used and the numerical integration technique. Other integration techniques, with the exception of an algebraic inversion of the centered differences used to create the dashed curve in Fig. 3.4, give similar results. In contrast to unperturbed initial conditions, in the perturbed cases the integration fails to correct itself; this emphasizes the fact that the forcing and the SST are completely decoupled in this case.

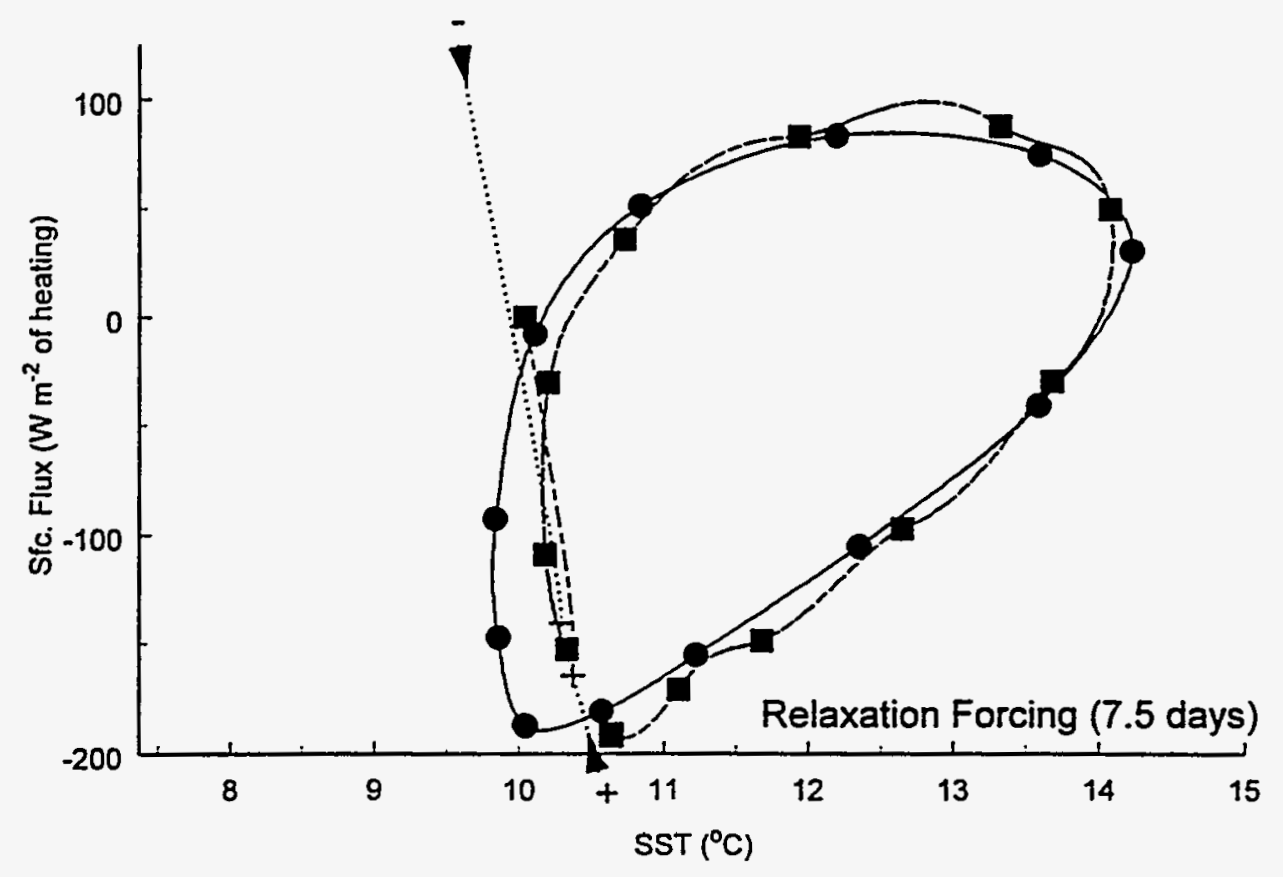

Figure 3.8 As in Fig. 3.7 for pure relaxation forcing with a time constant of (7.5 days) $)^{-1}$.

With unperturbed initial conditions, the initial implied flux from relaxation forcing, which uses Eq. 2 (Fig. 3.8), is zero, and this is quickly corrected (which explains the loop in 
USDOE Grant No. DE-FG03-93ER61646

Final Report

Page 24

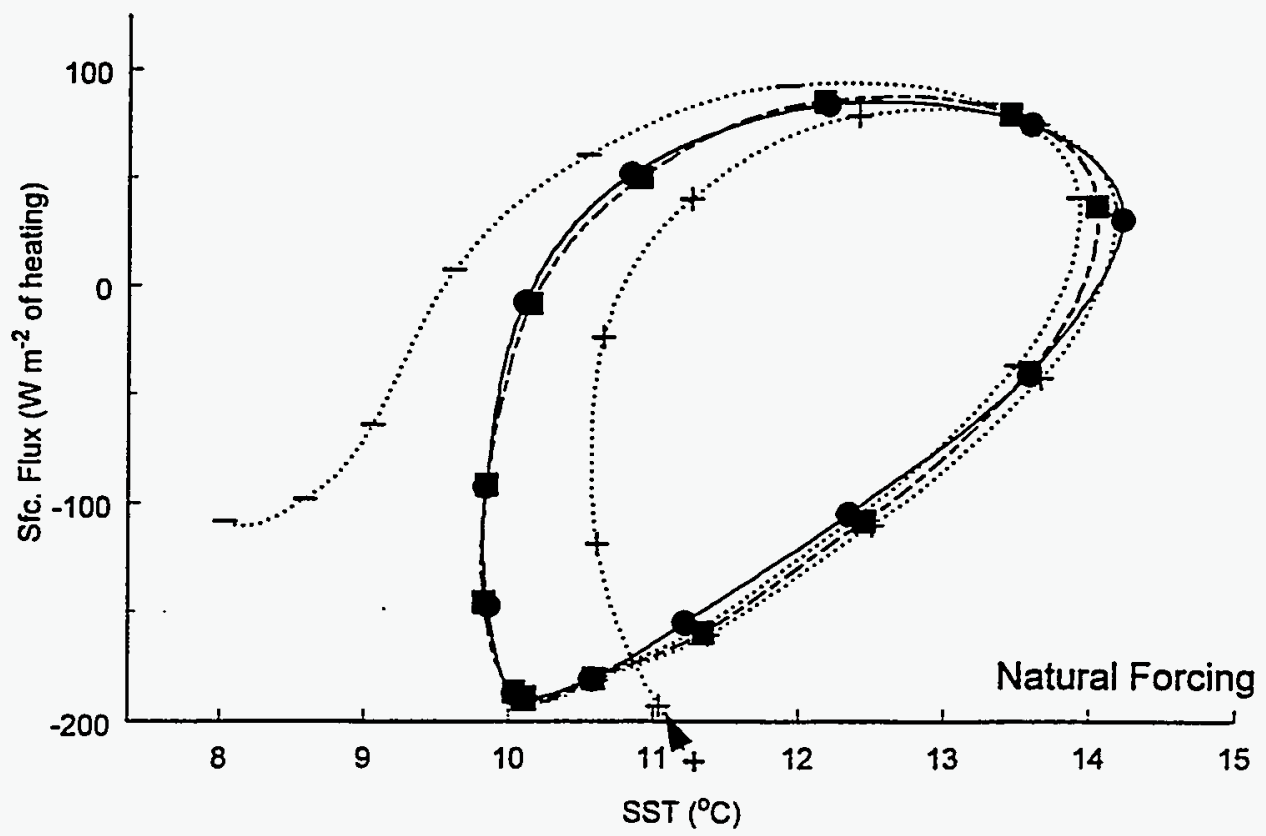

Figure 3.9 As in Fig. $3.7 \& 3.8$ with natural boundary conditions from (6), (7), and (13).

the dashed curve in Fig. 3.8). Further, the perturbed initial conditions are also corrected rather quickly, and the two dotted curves overly the dashed curve in Fig. 3.8 after about the beginning of March. For the rest of the year, however, the relaxation forcing reacts too quickly to SST changes and causes the implied fluxes to wander; the resulting behavior of the partial phase diagram resembles a drunkard's walk about the observations. In addition, the phase of the SST for the individual months is not reproduced very well. These results are also insensitive to integration technique.

Under mixed boundary conditions, using (6), (7), and (13) to calculate the surface flux, the model reproduces the observations quite well for the unperturbed case and relaxes smoothly toward the observations when the initial conditions are perturbed (Fig. 3.9). The monthly phase of the SST is somewhat better than in the pure relaxation case as well. Visually, the results in Fig. 3.9 resemble a combination of the other two cases, retaining the favorable aspects of each and eliminating the objectionable aspects. 
USDOE Grant No. DE-FG03-93ER61646

Final Report

Page 25

\section{iii. Discussion}

Because the wind-driven ocean circulation has long been a matter of keen interest to the oceanography community, wind-stress climatologies have a richer history than thermodynamic forcing climatologies. As mentioned in the Introduction, it is also easier to use wind-stress forcing: it is simply a flux boundary condition on the momentum equations. There are, however, three remarks that are relevant to the discussion here.

First, it is crucial to emphasize that the use of the thermodynamic forcing set discussed in Sections $2 \& 3$ requires including the wind speed from this same set. This is due to the calculations of $T_{E f f}$ and $q_{E f f}$. For consistency, the wind stress and wind speeds should ideally be developed from the same climatology.

Second, some OGCMs, particularly those including surface mixed layers (e.g., Bleck et $a l ., 1989$; Oberhuber, 1993), require not only the wind stresses as forcing for the momentum equations but also some quantity related to the magnitude of the surface wind stirring, such as the friction velocity. While this could be calculated from the wind speed by specifying a drag coefficient, it can also be calculated more explicitly using the subgrid, sub-monthly correlations of the wind observations (the pseudo-stress) and the wind- and stability-dependent density and drag coefficients used in the thermodynamic calculations above.

Third, while the relaxation of the SST to atmospheric conditions is not directly mimicked by the ocean currents, there are circumstances in which the input of momentum by the wind into the ocean is affected by the presence of strong currents, such as those in the Gulf Stream or in the vicinity of strong mesoscale circulations (e.g., Rooth and Xie, 1992). A first-order correction for this effect can be derived in a manner analogous to the thermodynamic forcing discussed here 
USDOE Grant No. DE-FG03-93ER61646

Final Report

Page 26

by correcting the stresses ( $\tau$ ) for the current:

$$
\left.\tau_{(x, y) E f f} \equiv \tau_{(x, y)}-\frac{\sqrt{\left(\tau_{x}^{2}+\tau_{y}^{2}\right)}}{u_{*}{ }^{2}}\right]\left(u^{2}, v^{2}\right),
$$

where the term involving the radical is the density times the drag coefficient calculated consistently with the stresses, $u_{*}$ is the friction velocity calculated as discussed above, and $(u, v)$ are the zonal and meridional currents reckoned in the same direction as the winds (i.e., eastward and northward positive, respectively). The effective wind stress is thus reduced by a current in the same direction. This neglects second-order effects, involving wind/current cross products; but these are expected to be small.

As ocean circulation modelers take advantage of modern supercomputer power, the use of complete physics that include especially the budgets of salt and heat is becoming more routine. Eventually it will be possible to calculate the surface boundary conditions for these budgets with the use of coupled atmosphere-ocean models. Further, it is likely that there will always be motivation for stand-alone OGCM calculations no matter the computing power available, and, despite the availability of simplified atmospheric models (e.g., Kleeman and Power, 1995), the use of atmospheric climatologies for forcing these calculations will continue. Meanwhile, formulating these surface boundary conditions for stand-alone OGCMs requires attention to the behavior of the real ocean and its response to atmospheric forcing.

This discussion has been concerned with these surface boundary conditions. Expedient forcing methods used in the past for wind-driven circulation studies that include these budgets-specifying the surface flux or adopting an approach that relaxes the model toward observed SST and salinity - have significant limitations, both mathematically and physically. It has been shown here that significant errors can be introduced by using these approaches. The 
USDOE Grant No. DE-FG03-93ER61646

Final Report

Page 27

use of the partial phase diagrams introduced in Section 3 reveals consistent amplitude and phase errors associated with attempts to replicate the annual cycle of the SST using flux or relaxation forcing. More specifically:

(1) The use of marine climatologies such as the COADS provides a physically consistent set of forcing conditions, provided (a) there is a method available for estimating the surface radiation budget and the precipitation and (b) sub-grid correlations are included in the forcing conditions. As shown here, neglecting the latter can bias the net surface heat flux by as much as 40-50 W $\mathrm{m}^{-2}$.

(2) Neglecting to calculate the saturation mixing ratio associated with the model SST, although attractive from a computational standpoint, can lead to significant surface flux errors that introduce spurious positive feedback into the calculation.

(3) The simplest expedient for specifying the surface forcing of the heat and salt budgets, specifying the fluxes, decouples the calculation from the forcing and eliminates any way for the model to correct itself when perturbed by other processes.

(4) Relaxation conditions, using specified surface climatologies not only bias a calculation toward a particular climatic state but also introduce phase errors into the calculation itself.

For these reasons, the mixed boundary conditions discussed here produce more realistic model behavior despite their relatively greater complexity and computational demand. An additional attraction of this approach is that OGCMs formulated to use it are automatically ready for integration into a coupled model.

\section{b. Scales of forcing}


USDOE Grant No. DE-FG03-93ER61646

Final Report

Page 28

As shown here, surface forcing of an ocean model is most realistically formulated as a combination of flux and relaxation boundary conditions at the surface. If an ocean model is used in isolation, it is also necessary to decide what time and space resolution is appropriate for the specification of the atmospheric quantities of the forcing. In a coupled model, differences in time steps between the atmospheric and oceanic components raises similar questions about how to average (or interpolate) the atmospheric state variables for the oceanic calculation.

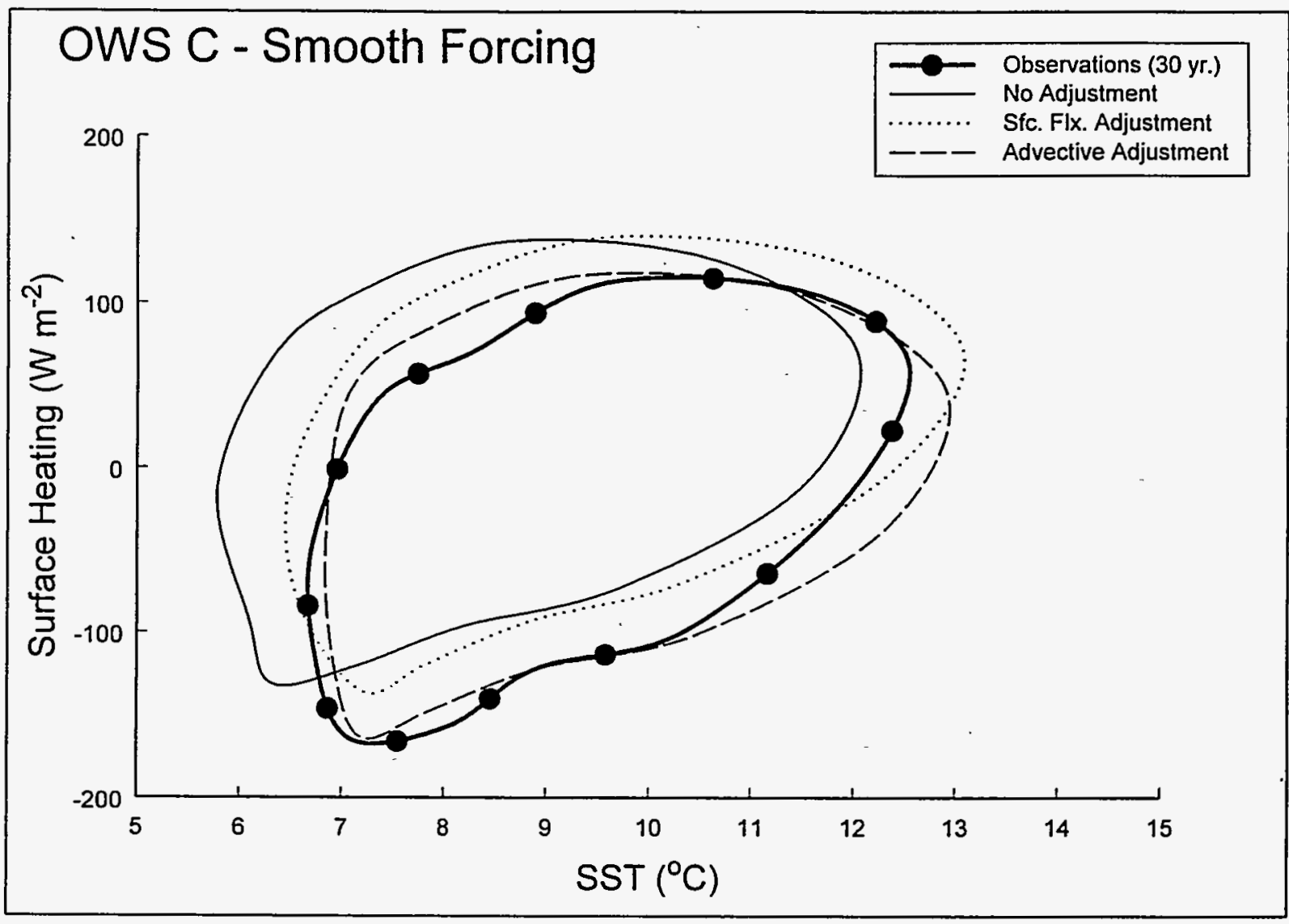

Fig. 3.10 One-dimensional MICOM integrations showing effects of correcting for advection. the "advective adjustment" is used in subsequent results.

Most ocean circulation calculations have used monthly-averaged climatological forcing interpolated onto the oceanic time step and grid. However, it is clear that there is substantial 
USDOE Grant No. DE-FG03-93ER61646

Final Report

Page 29

atmospheric variability on shorter time scales. It is therefore useful to assess how important this shorter term variability is for the ocean. This is done here with a one-dimensional version of the MICOM code; as in the previous section, conditions appropriate for the North Atlantic are examined. The effects of advection in this section are included by simply using the annual imbalance of the observed net surface heating to correct the SST integration at each time step. Correcting the fluxes themselves (by increasing, say, the solar heating) results not only in poorly calculated surface fluxes but it also affects the mixed-layer behavior, because mixed-layer entrainment depends strongly on the surface forcing. As shown in Fig. 3.10, an advective adjustment correction, in which the surface flux imbalance is spread throughout the mixed layer

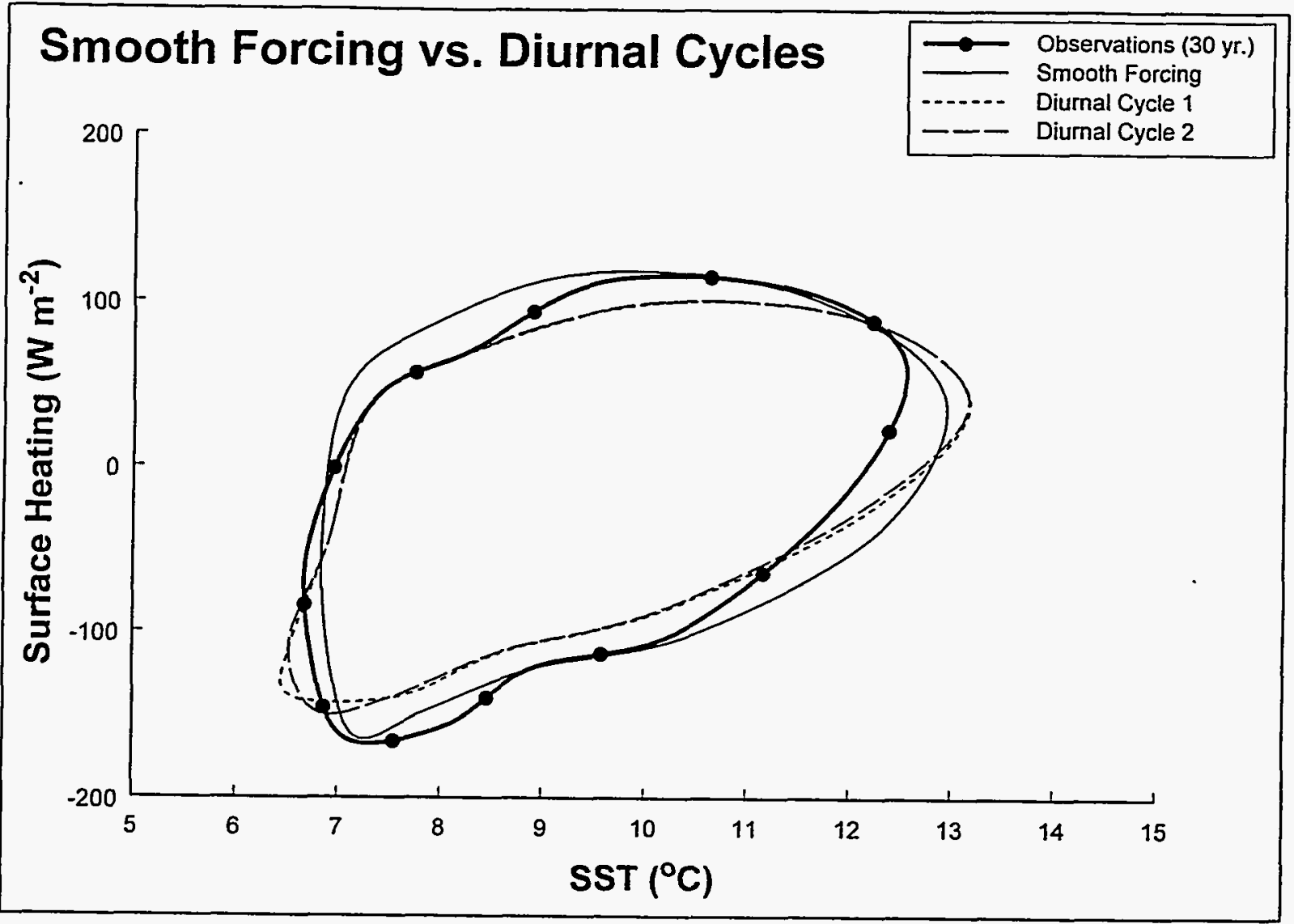

Figure 3.11. One-dimensional MICOM integrations without (solid) and with two formulations of a diurnal cycle of solar heating. Springtime behavior is improved, but overall behavior is not. 
USDOE Grant No. DE-FG03-93ER61646

Final Report

Page 30

as if it were advection, provides a more realistic result. There are still, however, large differences between the model results and the observations.

These results were obtained using the (interpolated) monthly averaged forcing developed from COADS. This forcing is quite smooth compared to nature; in particular, small-time-scale variability is missing. The most obvious components of this are the diurnal solar cycle and synoptic-scale winds.

Including the diurnal cycle is relatively straightforward and has dramatic consequences for the behavior of the mixed-layer depth. This is because each day there is a period of cooling (at night) with entrainment and a period of heating without it. Consequently, the mixed layer never has a chance to become as deep in the winter as it does without the diurnal cycle. Mixedlayer depths are there much more realistic with the dirunal cycle. However, including the diurnal cycle does not really improve the phase diagram significantly. This is shown in Fig. 3.11, which uses two "diurnal cycles". Because the MICOM time step in these calculations is $1 / 15$ th of a day, a quasi-gaussian solar heating component can be distributed over either 7 or 8 time steps conveniently. Fig. 3.11 shows that there is little difference in the choice of the length of the period of solar heating, in this context. One significant improvement in model behavior, compared to the smoothed, monthly-averaged forcing, occursdfuring the sprintime months, when the seasonal thermocline is forming. This suggests that including the dirunal cycle is important for simulating correctly the subduction processes associated with the annual cycle of the mixed layer. 
USDOE Grant No. DE-FG03-93ER61646

Final Report

Page 31

OWS C Annual Cycle

30-Year Standard Deviations

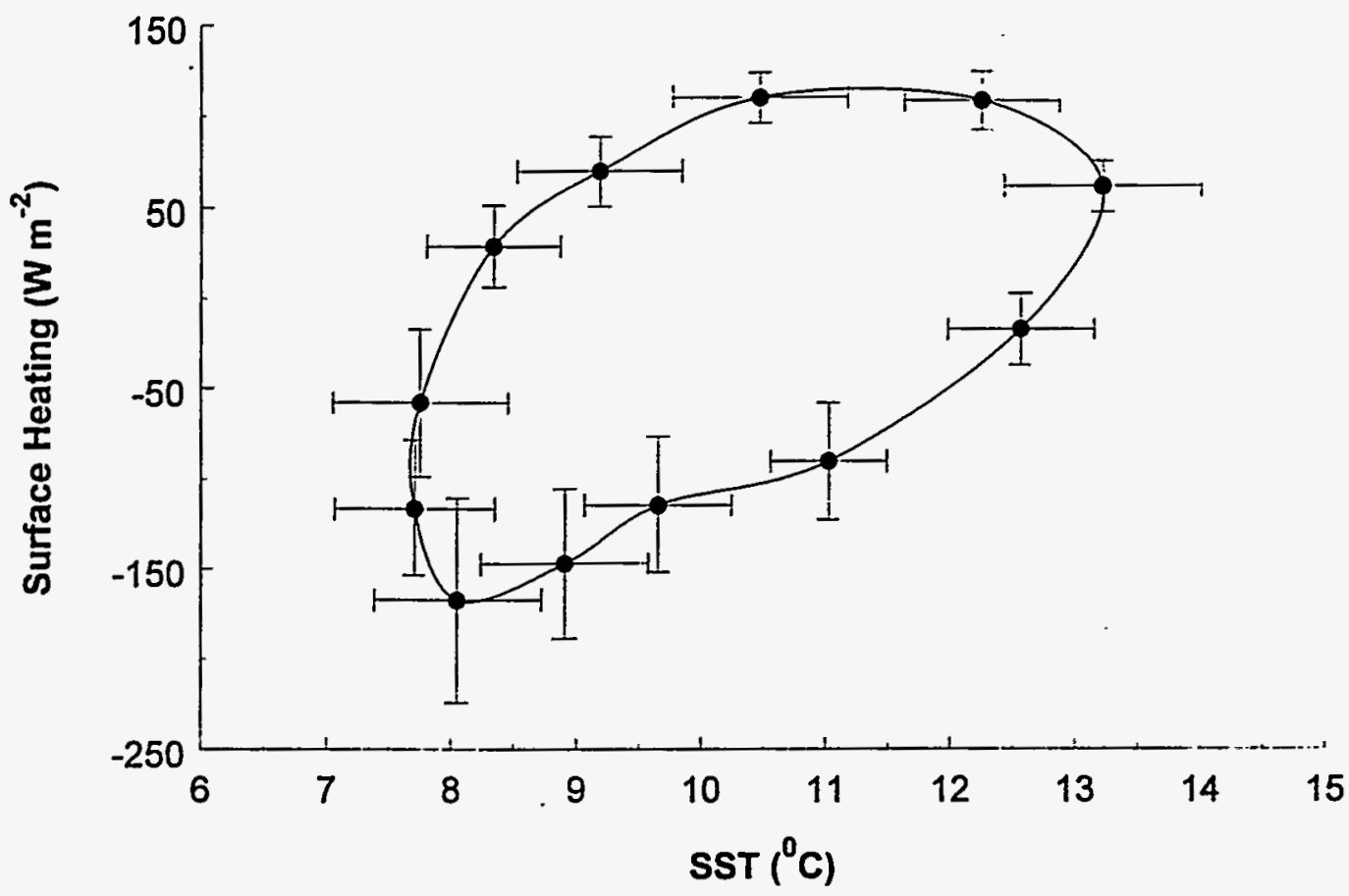

Figure 3.12 Year-to-year variations in COADS climatology at OWS C, in terms of standard deviations of SST and surface heating.

Just as the solar heating is not smooth on the scale of months, so is the wind forcing intermittant, due to synoptic-scale storms. The observed phase diagrams shown here, for OWS $\mathrm{C}$ and for the other latitides in Figs. 3.6, are interpolated from 30-year averages of monthlyaveraged data, and the smoothed forcing is also in this form. Year-to-year variations within this 30-year climatology are significant (Fig. 3.12).

In order to test the effects of synoptic-scale variability on the MICOM, the onedimensional version was integrated using the smoothed, climatological forcing with zero-mean perturbations superimposed. The synoptic-scale wind perturbations used here were created using a stochastic red-noise model that incorporates the statistics of the observed wind variability and 
USDOE Grant No. DE-FG03-93ER61646

Final Report

Page 32
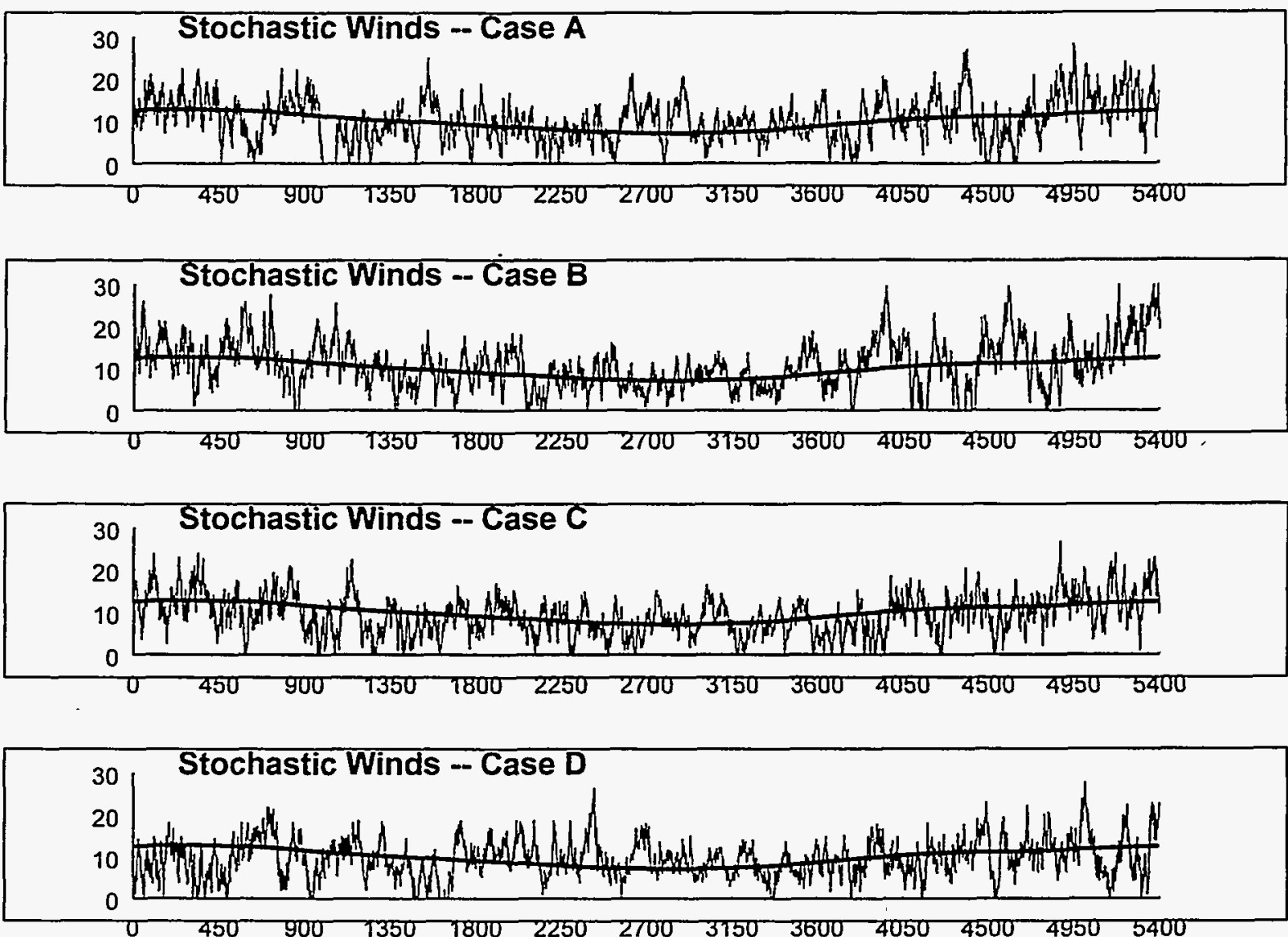

Figure 3.13. Four realizations of the stochastic wind model (thin lines) superimposed on the climatological wind speed (thick line). The abscissa is the model time step.

produces wind time series with the same spectral characteristics as the observed winds. A random-number generator is used in the generation of each realization; the calculations shown here depict years 32 of separately initialized realizations (and averages of 30 such integrations). In each realization, the random-number generator was seeded with the system clock time. Fig. 3.13 shows time series wind speed of four typical realizatons (at 15 time steps per day, there are 5400 time steps in the 360 -day year of the model). The thick line represents the climatology. 


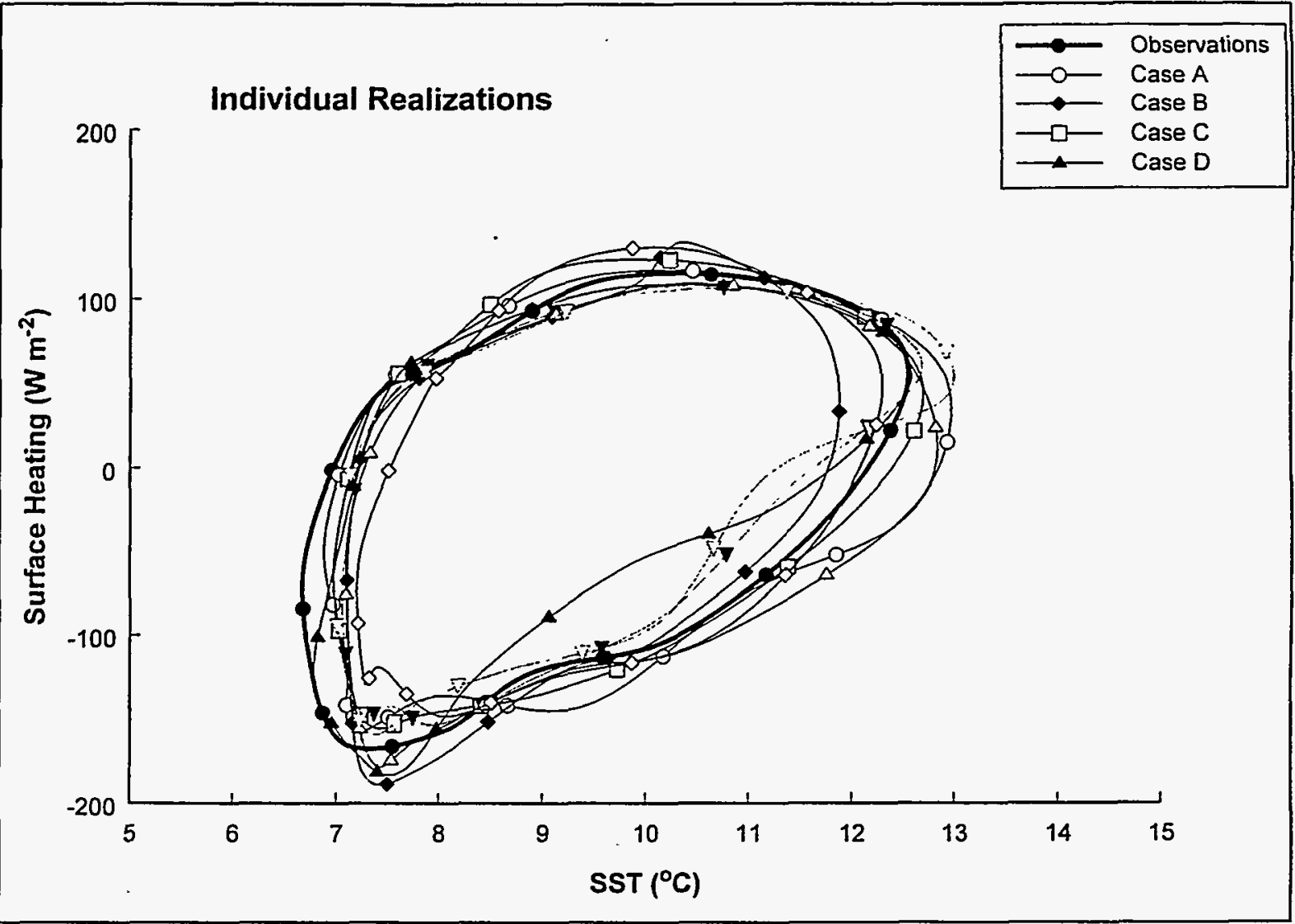

Figure 3.14. Phase diagrams from several one-dimensional MICOM integrations with different stochastic wind realizations. Labeled cases correspond to winds for year 32 in Fig. 3.14 .

Fig. 3.14 shows results from several individual stochastic realizations. The four cases labeled correspond to those of Fig. 3.13 (which shows year 32 winds; Fig. 3.14 shows year 32 phase diagrams); several others are shown as well. The results vary from realization-to-realization in much the way that the observations appear to (Fig. 3.12). In the phase plots of Fig. 3.14, the curve between about November and February should be ignored, as they are interpolated across non-continuous data). That is, year-to-year variations of the model integrations forced by synoptically varying winds represent the observed phase diagrams 


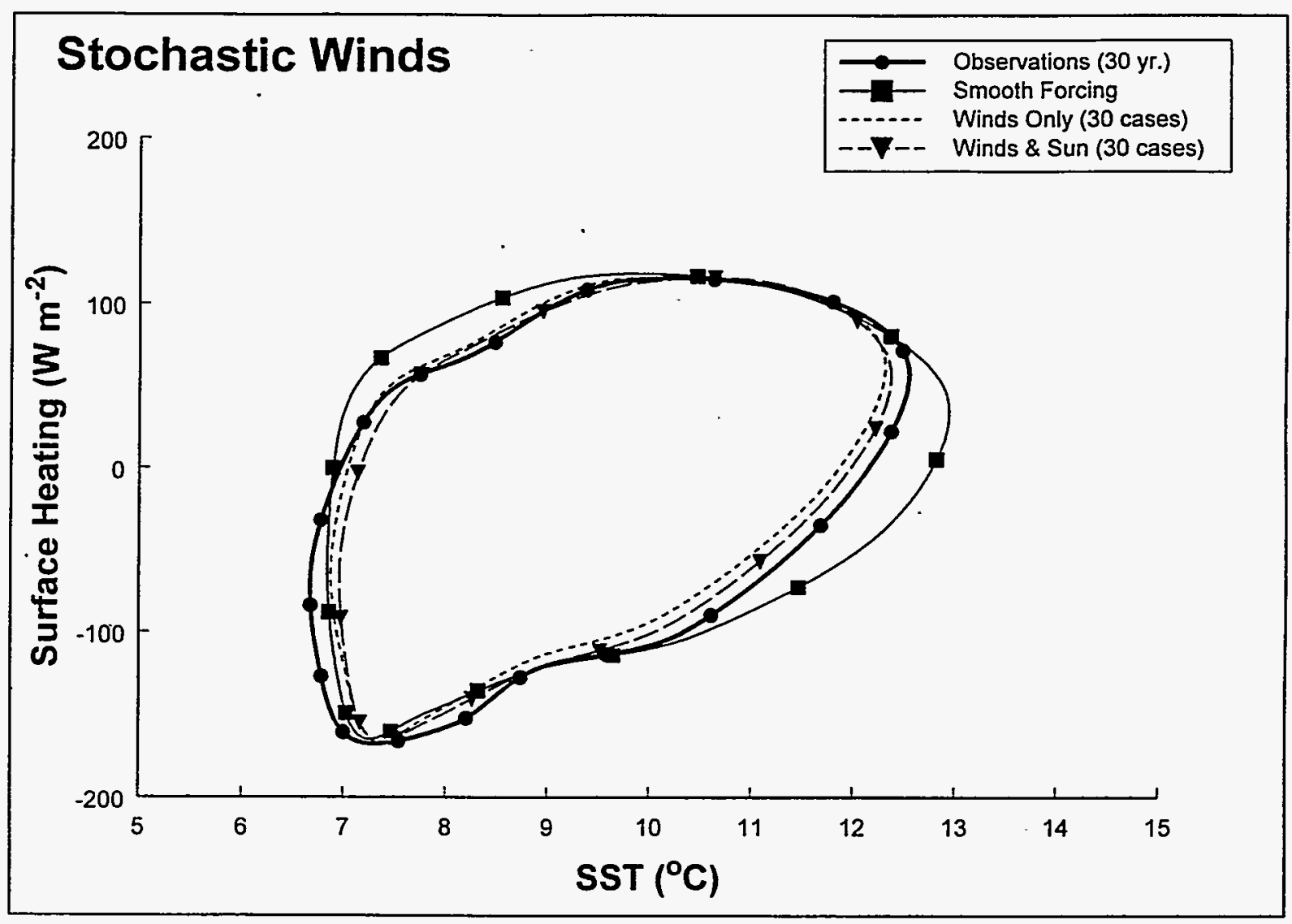

Figure 3.15. Averages of 30 realizations of 32-year integrations of stochastic wind forcing, with and without a diurnal cycle, compared with observations and-results of smoothed forcing.

more realistically than does the result of smoothed forcing. Moreover, when 30 such realizations are averaged, they converge to the climatology (Fig. 3.15), at least as much as might be expected, given that the "advective" component of these simulations has no annual variability. Although the difference associated with including the diurnal cycle in Fig. 3.15 is small, the diurnal cycle still has a dramatic effect on the mixed-layer depths in the model, and it needs to be included to make this component of the simulation realistic. 
USDOE Grant No. DE-FG03-93ER61646

Final Report

Page 35

\section{MICOM Results: Oceanic Responses to Global Warming}

Ms. Horsfall began her dissertation research in 1991 and successfully defended her results in the spring of 1996. The project involved using the MICOM in two forms to investigate the response of oceanic processes, particularly subduction on the time scale of the annual cycle, to changes in atmospheric forcing. The following discussion summarizes salient aspects of these results, using figures copied from her dissertation. Further details are available in the dissertation (Horsfall, 1996); some of these results were discussed in Horsfall et al. (1994a,b, 1997).
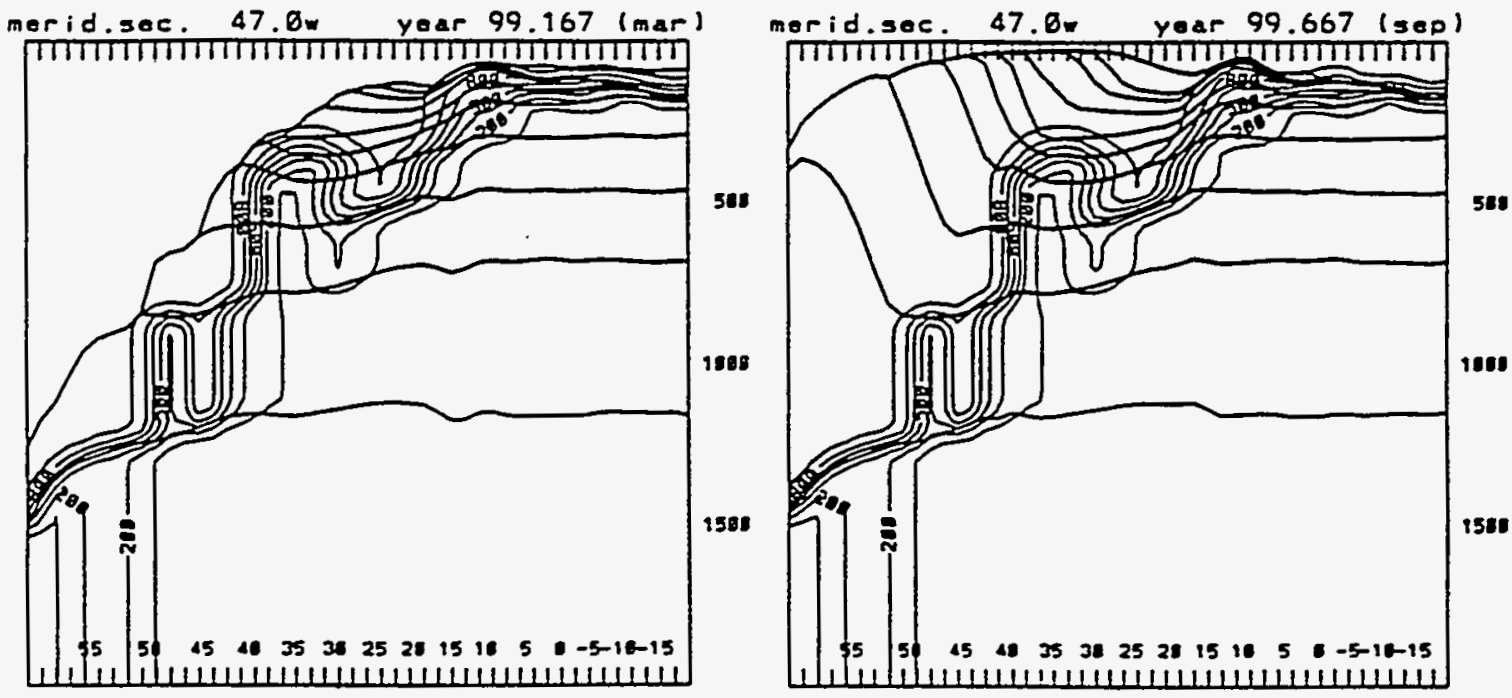

Figure 4.1a. (left) Meridional cross-section, at a longitude corresponding to approximately $47^{\circ} \mathrm{W}$, for the control run in March of year 99 . Model layers and contours of passive tracer $(\% \times 10)$ are shown. This deep mixed layer contrasts to the September cross-section in Fig. $4.1 \mathrm{~b}$, and shows how the annual cycle of the mixed layer contributes to subduction. $b$. (right) September cross-section. 
USDOE Grant No. DE-FG03-93ER61646

Final Report

Page 36

\section{a. Sector model experiments}

The MICOM was originally developed in a rectangular-basin, no-topography form, as discussed in Bleck et al. (1989). Because of its computational efficiency, this version of the MICOM was used to perform a variety of long-time integrations with various forcing perturbations.

Subduction associated with the annual cycle happens as water that is exposed to the atmosphere during the-winter season is left behind in the seasonal thermocline as the mixed layer retreats during the following spring/summer heating season. An example of this behavior, from the control integration, is shown by the north-south cross sections at $47^{\circ}$ Win Figs. 4.1. The top-

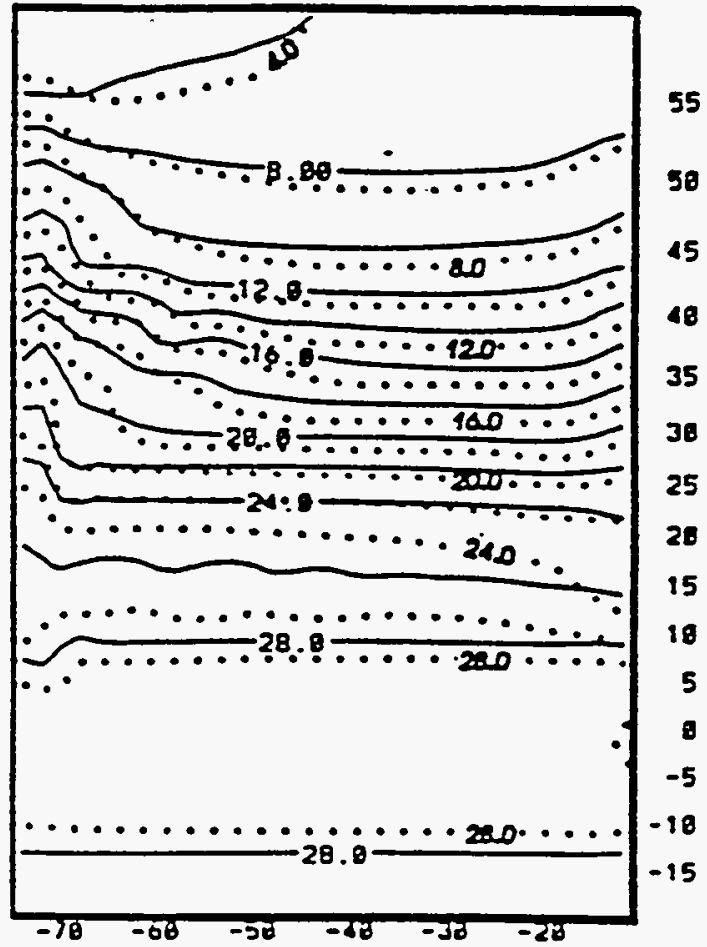

Figure 4.2. SST in March for the control run (dots) and the increased air temperature experiment (solid). most model layer shown is the base of the mixed layer, which extends to a depth of more than $1000 \mathrm{~m}$ in the northern part of the basin in March and then retreats to less than $50 \mathrm{~m}$ during September. The closed contours show concentrations of an artificial passive tracer in the model, the concentration of which tracer in maintained at $100 \%$ in the mixed layer. As seen in Figs. 4.1, after 50 years on model time, this tracer has significant concentrations in the deeper model layers. This is entirely due to the subduction process associated with the annual cycle, because, in this version of the MICOM, there is no diapycnal mixing (i.e., crossisopycnal transfer of water or other properties, 
USDOE Grant No. DE-FG03-93ER61646

Final Report

Page 37

the equivalent of vertical diffusion in the isopycnic framework).

The question that Ms. Horsfall addressed concerned the changes in this subduction that occur in response to changes in atmospheric forcing of the ocean. The sector model was spun up for 50 years as the control using observed climatological forcing (smoothed monthly means), and the experiments were each begun from year 50 control conditions and integrated for an additional 50 years. Experiments included increasing the downward radiative flux, the air temperature, the wind speed, the wind stress, and various combinations of these components of forcing. In the sector model experiments, the variations in surface forcing were not latitude/longitude dependent. The changes in forcing were chosen to represent approximately changes that might be expected to accompany greenhouse-induced global warming.
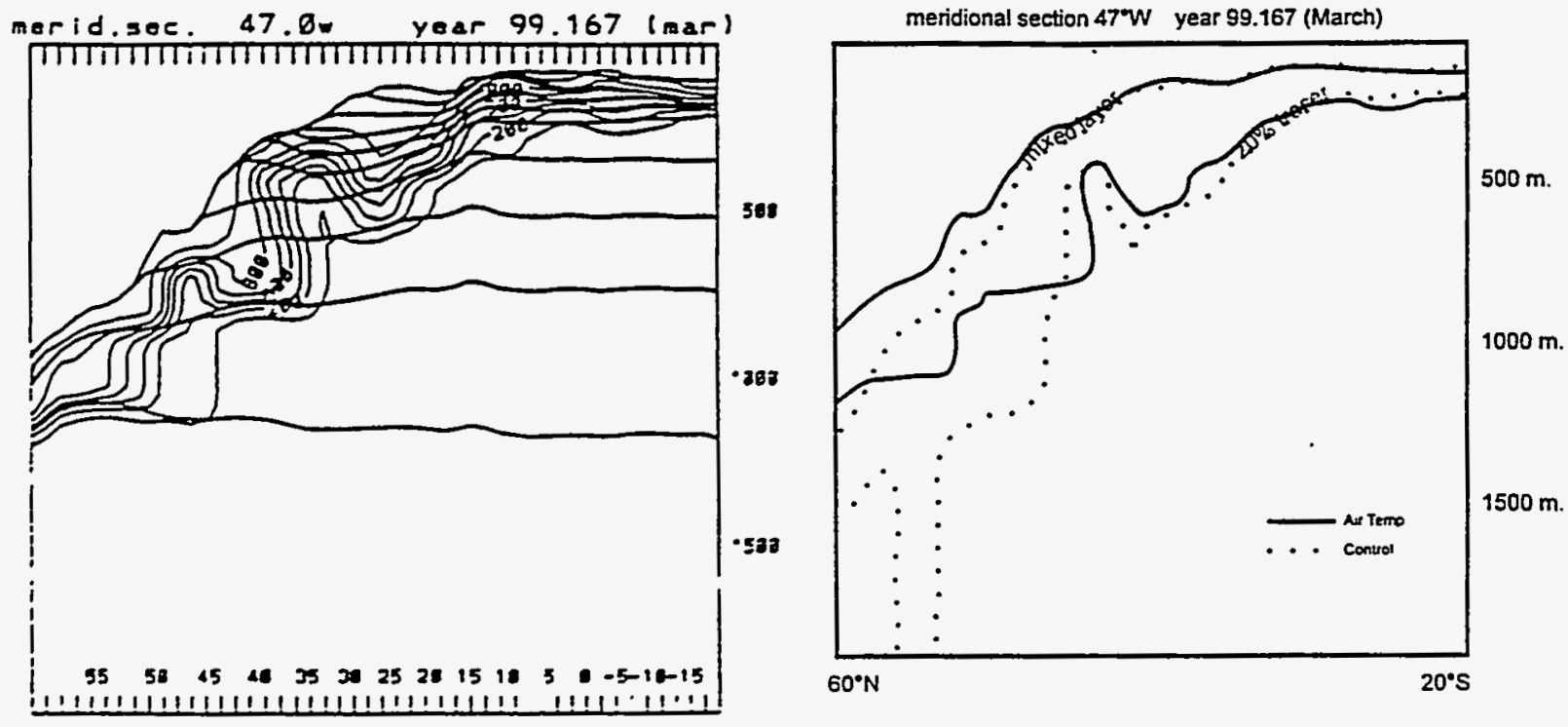

Figure 4.4. Mixed layer depth (upper) and 20\% Figure 4.3. March cross-section at $47^{\circ} \mathrm{W}$ fortracer penetration depth (lower), $47^{\circ} \mathrm{W}$ in the increased air temperature experiment. March. 
USDOE Grant No. DE-FG03-93ER61646

Final Report

Page 38

Fig. 4.2 compares SSTs over the basin for the control run and for the experiment that used increased air temperatures (by $3^{\circ} \mathrm{C}$ ) in the model forcing. "Global warming" is, in essence, defined as surface air temperature increases, so this represents the lowest-order experiment to test the model's response to potential effects of an increased greenhouse effect. As might be expected, SSTs are warmer for this experiment, because warmer air temperatures decrease upward turbulent heat fluxes (or, as discussed in Section 3 above, provide the model with a higher set of temperatures to relax toward). Fig. 4.3 is the $47^{\circ} \mathrm{W}$ March cross section for this experiment, and it shows that the mixed-layer depth during March is less and the tracer penetration is not as deep as in the control case. These effects are summarized in Fig. 4.4.

Of course, the fundamental result of increases in the greenhouse effect is to increase the downward infrared flux from the atmosphere to the surface, and the effect of this change in surface forcing was also tested by increasing the surface radiative heating by $5 \mathrm{~W} \mathrm{~m}^{-2}$. Unlike
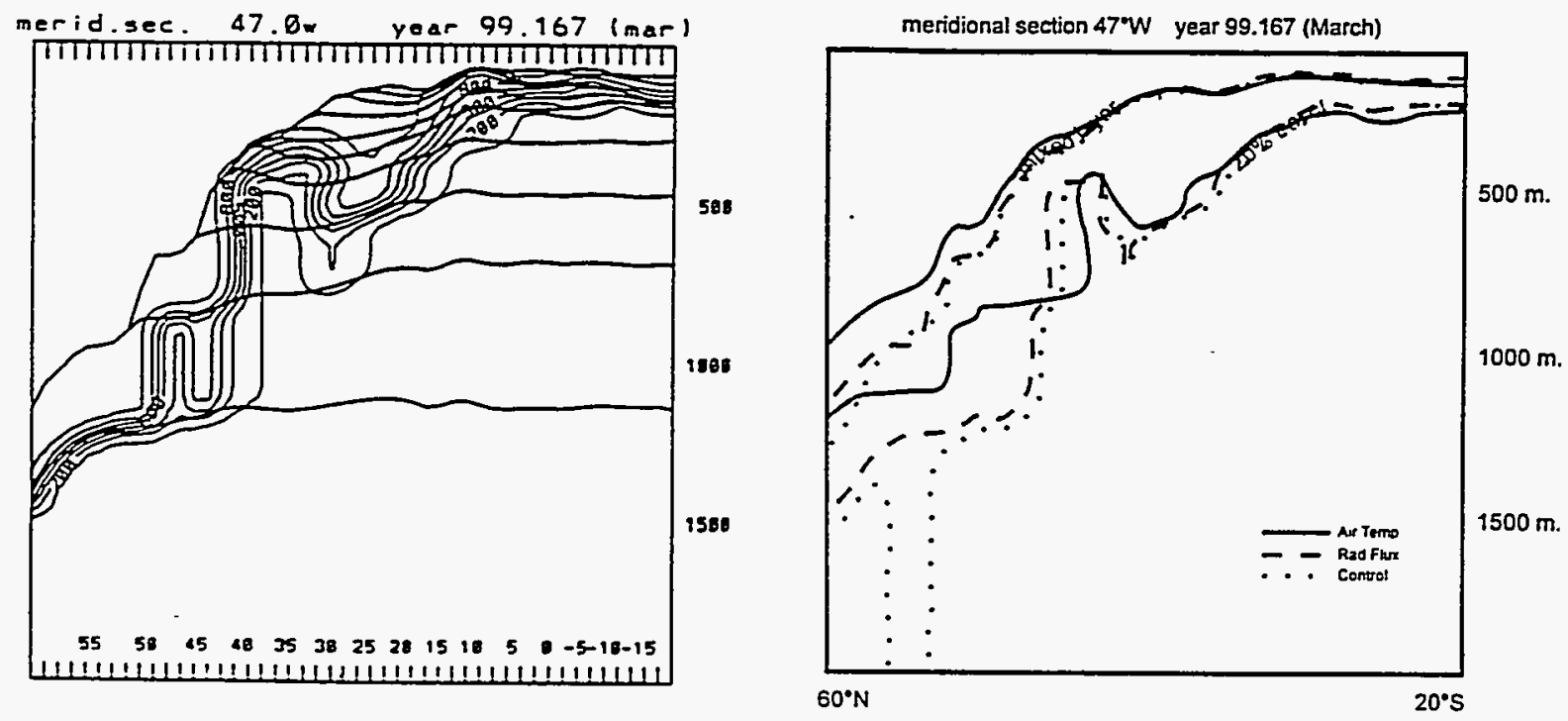

Figure 4.5. March cross-section for increasedFigure 4.6. Mixed layer depth (upper) and 20\% radiative flux experiment. tracer penetration depth, $47^{\circ} \mathrm{W}$, March. 
USDOE Grant No. DE-FG03-93ER61646

Final Report

Page 39

the changes in air temperature, this is a change in the surface flux (direct) component of surface forcing. Its effect is smaller than the effect of increased air temperature. This can be seen in Fig. 4.5 (the $47^{\circ} \mathrm{W}$ cross-section) and the summary of both experiments in Fig. 4.6.

Therefore, it can be concluded that the direct effect of global warming on subduction associated with the annual cycle of the mixed layer is to decrease the amount of subduction, and this implies that the feedback processes discussed in Section 1

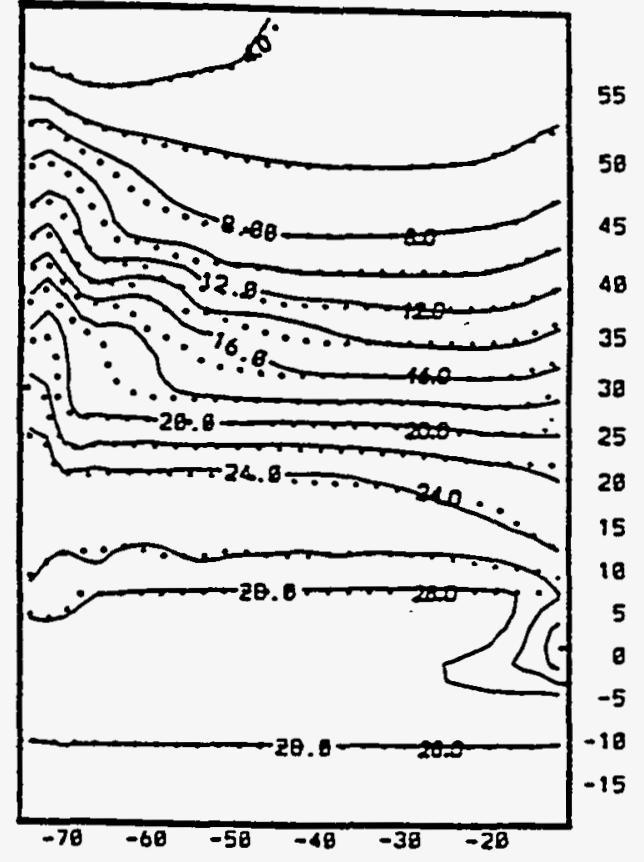

Figure 4.7. March SSTs for control (dotted) and increased wind stress experiments (solid). above are self-amplifying. Not only will less heat be sequestered in the seasonal thermocline, but sequestration of greenhouse gases will be decreases as well due to these processes.

However, it is unrealistic to expect that the only changes in the atmosphere associated with global warming will be increased surface radiative heating and increased air temperatures. Calculations using global climate models (e.g., Manabe and Wetherald, 1975; Manabe et al. 1991,1992 ) have also shown that the latitudinal distribution of air temperature changes (which is not included in the experiments discussed thus far) is expected to be accompanied by increased baroclinicity, resulting in stronger winds in mid-latitudes. This effect is felt by the MICOM through two mechanisms: the increased wind stress would tend to accelerate the oceanic circulation, and the increased wind speeds would increase turbulent mixing in the upper ocean. Consequently, it may be the case that these effects could offset some of the thermodynamic changes shown above. 
USDOE Grant No. DE-FG03-93ER61646

Final Report

Page 40

This was tested in two experiments, one in which wind stresses were increased by $50 \%$ over the basin (with no increase in the wind speed where it appears in mechanical and turbulent mixing forcing terms) and one in which the wind speed was increased by $50 \%$ (with no change in the wind stress).

Fig. 4.7 compares SSTs for the control and increased wind stress experiments. Although the circulation was increased, changes in the SST patterns are small and confined to advective affects (most evident in the western boundary current and in the basin's southeast corner). The $47^{\circ} \mathrm{W}$ March cross-section (Fig. 4.8) is not significantly different from the control case (Fig. 4.1a) nor is the summary section of mixed-layer depth (not shown).

When the wind speed is increased, however, the changes are dramatic. SSTs (Fig. 4.9) are generally lower than in the control and the cross-sections reveal deeper mixed layers and
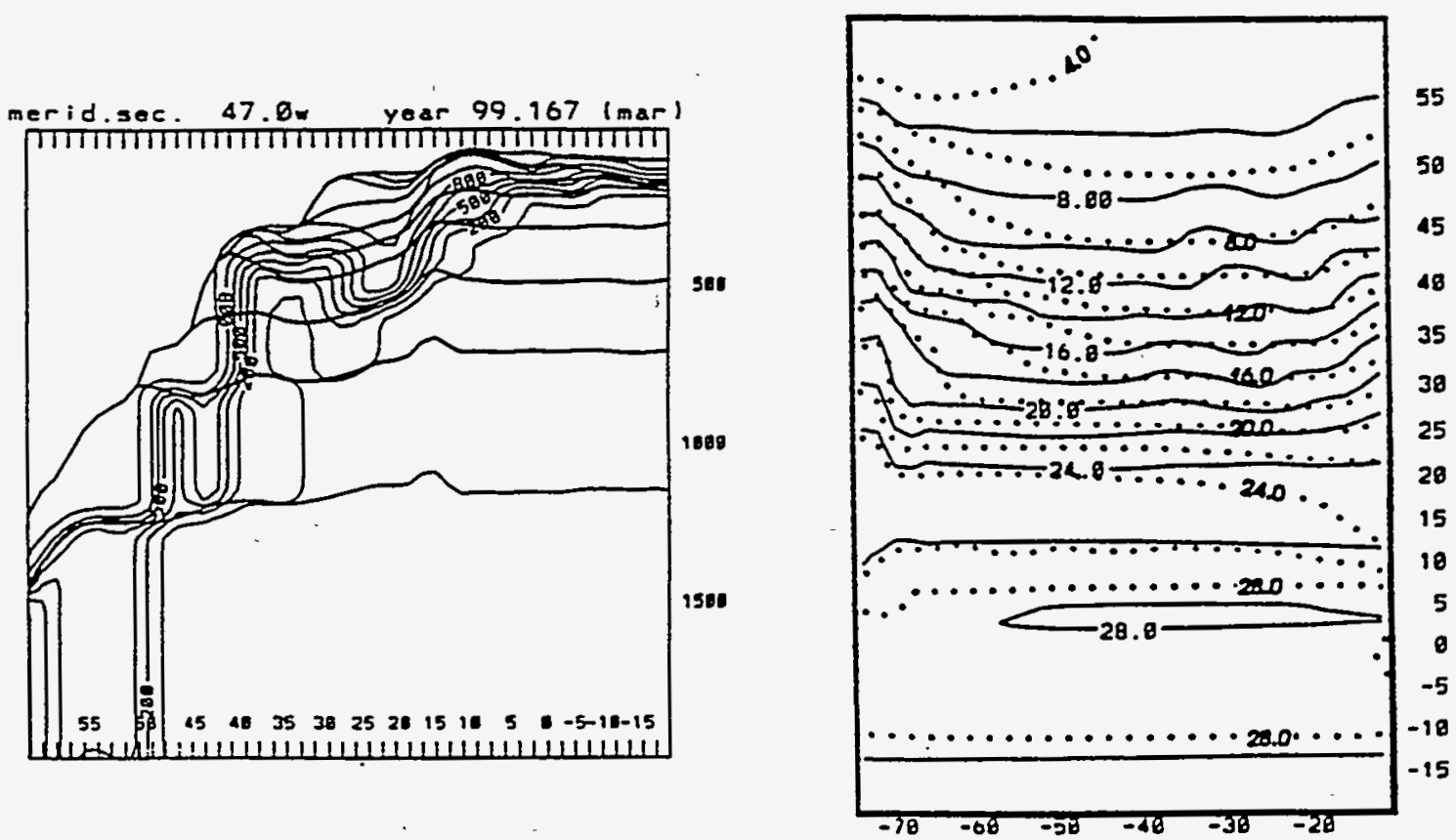

Figure 4.8 March cross-section for increasedFigure 4.9 March SSTs for control (dotted) and wind stress experiment. increased wind speed experiment (solid). 
USDOE Grant No. DE-FG03-93ER61646

Final Report

Page 4I
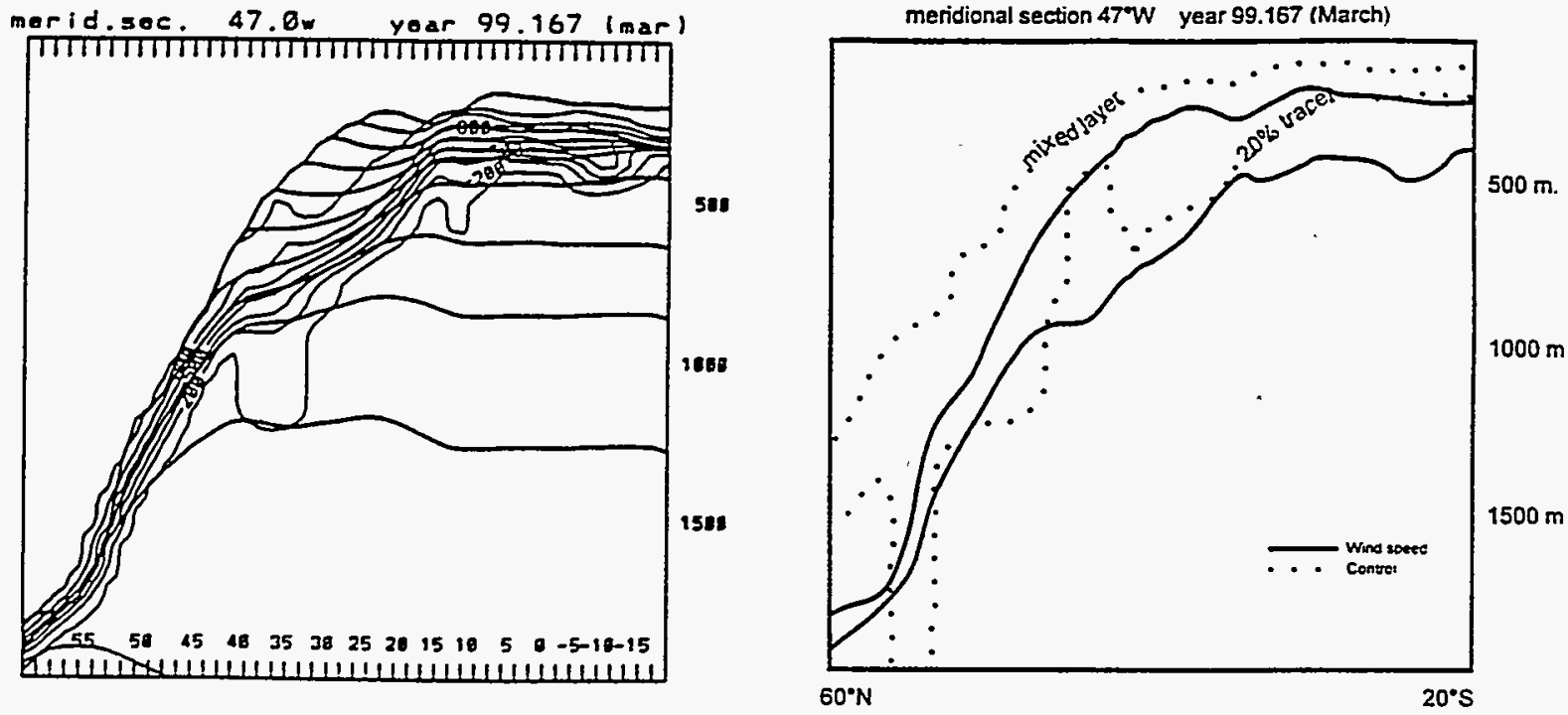

Figure 4.11 March mixed layer depth (upper) Figure 4.10 March cross-section for increase and 20\% tracer penetration (lower), increased wind speed experiment. wind speed and control.

greater tracer penetration (Figs. $4.10 \& 4.11$ ). Not surprisingly, the effect of consistent increases in wind (i.e., the combination of these two separate experiments) is dominated by the wind speed effects (Figs. $4.12 \& 4.13$ ).

These results show that the effects of increasing baroclinicity counteract the effects of rising temperatures, as far as oceanic subduction is concerned. The specific amounts of the increases used in the experiments here are somewhat arbitrary (the only way to make them self consistent would be to use atmospheric GCM results, which not consistent across model platforms), so it is not possible to quantify precisely the degree to which the effects counteract, but a qualitative measure can be found by a combined experiment, in which air temperatures are increased in combination with the wind speed and stress increases. Results from such an experiment are shown in Figs 4.14 and 4.15. 
USDOE Grant No. DE-FG03-93ER61646

Final Report

Page 42
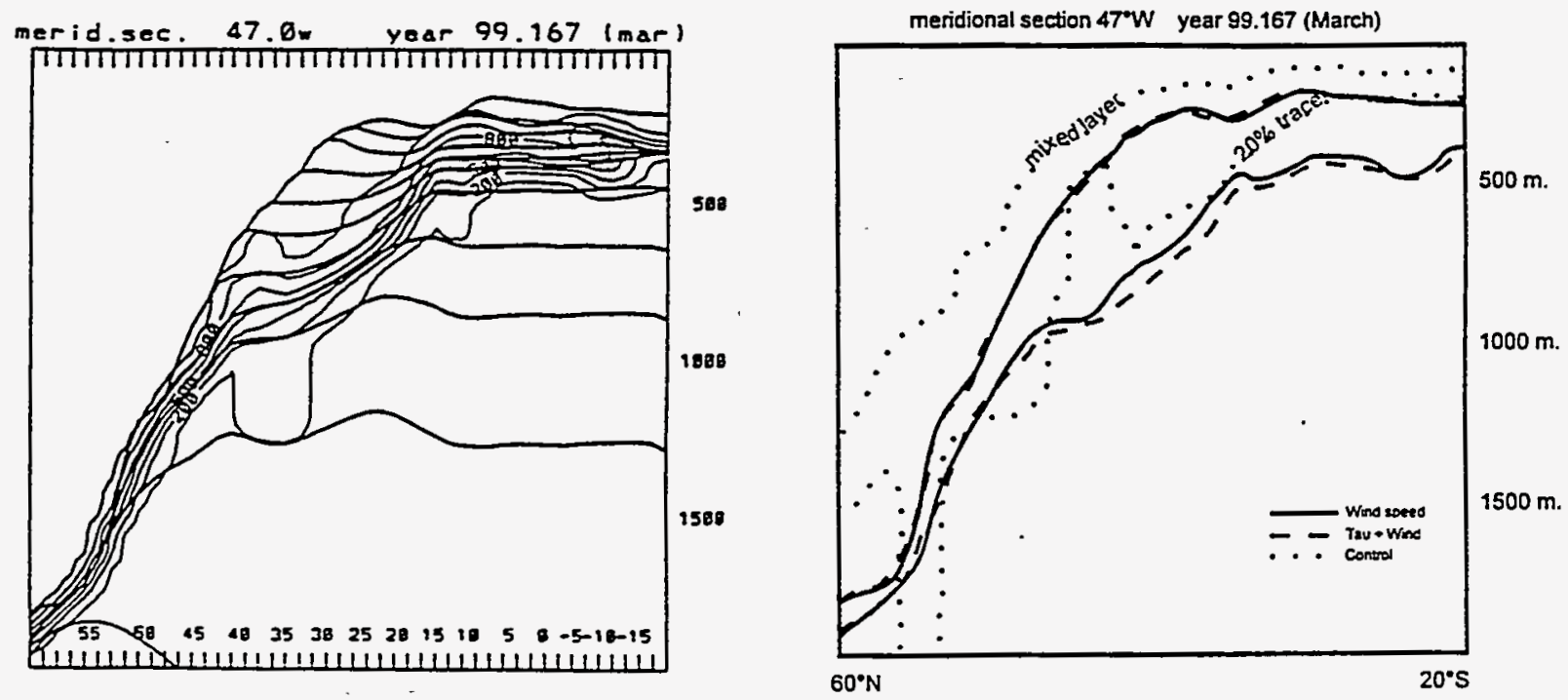

Figure 4.13 March mixed layer depth (upper)

Figure 4.12 March cross-section for increasednd 20\% tracer penetration depth (lower), winds experiment. increased winds, wind speed, and control.

With the exception of the smaller-scale variations, the cross-section for the various individual experiments (Figs 4.1a, 4.3,4.10) that compares most closely with this combined case (Fig. 4.14) is the control case (Fig. 4.1a). This is borne out in the summary of mixed-layer depth and tracer penetration shown in Fig. 4.15.

The various experiments (including one not discussed here that used increased vertical resolution in the model) with the sector version of the MICOM are summarized in Figs. 4.16. Tracer accumulation below the mixed layer, which is a measure of total subduction over the basin, shows how the effects of wind speed increases and air temperature increases (as well as downward radiative flux increases, tent to counteract each other. The Wind23 experiment used increased wind speed forcing with doubled vertical resolution in the model, and the difference 

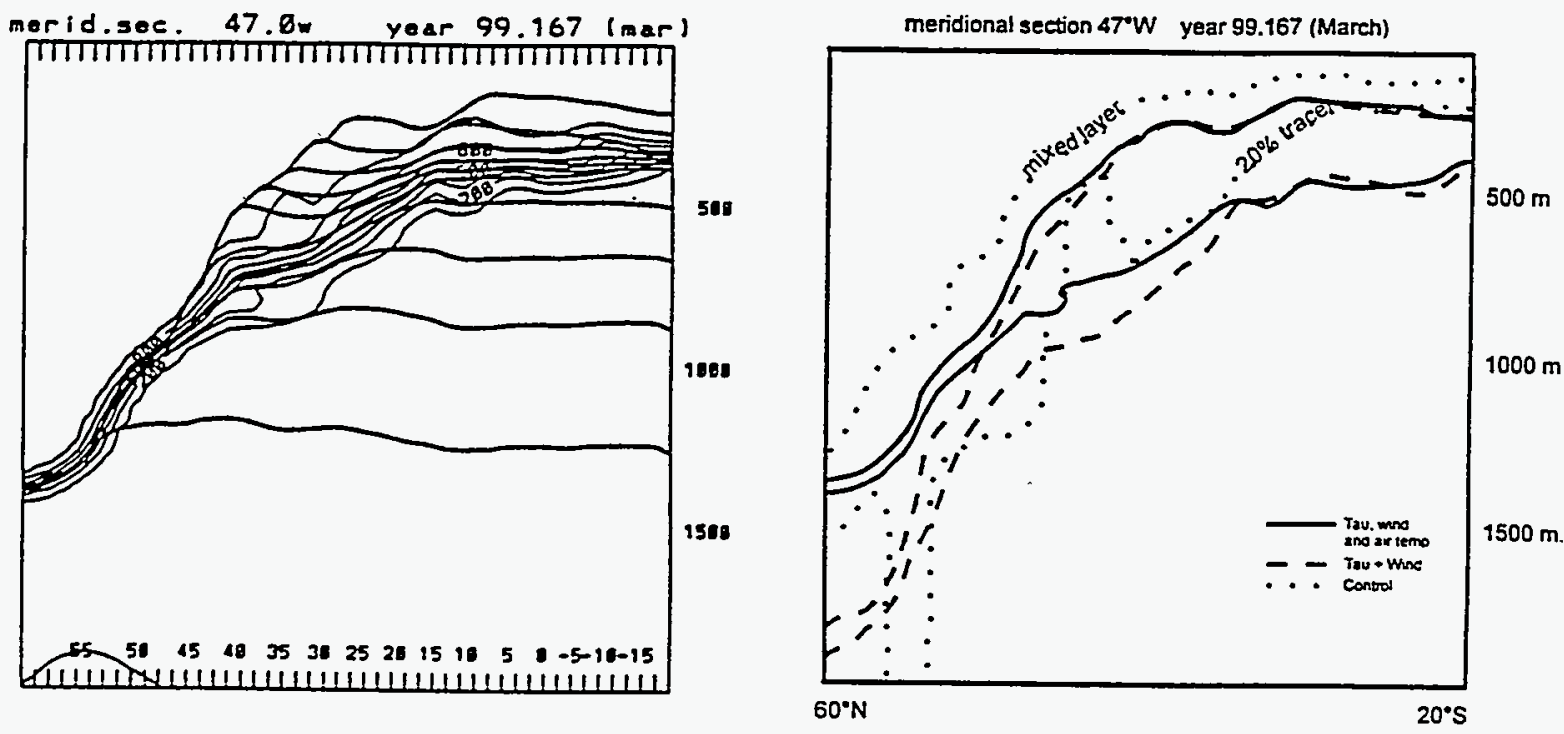

Figure 4.15 March mixed layer depth (upper) Figure 4.14 March cross-section for increase and 20\% tracer penetration (lower), increased air temperature, and winds. winds, increased winds \& temperatures, control.

between it and the Windspd*1.5 experiment is a measure of the noise or uncertainty level of these experiments; in terms of this tracer, it would appear that changes of the order of $5 \%$ must not be considered significant. Therefore, while the individual experiments (e.g., Airtmp $+3 ;$ taw) show significant changes in overall subduction, the combined one does not.

Consequently, it must be concluded that the overall effect on the ocean of potential changes of atmospheric forcing associated with global warming is ambiguous at best. These results were tested in Ms. Horsfall's dissertation using a more complete version of MICOM. 
USDOE Grant No. DE-FG03-93ER61646

Final Report

Page 44

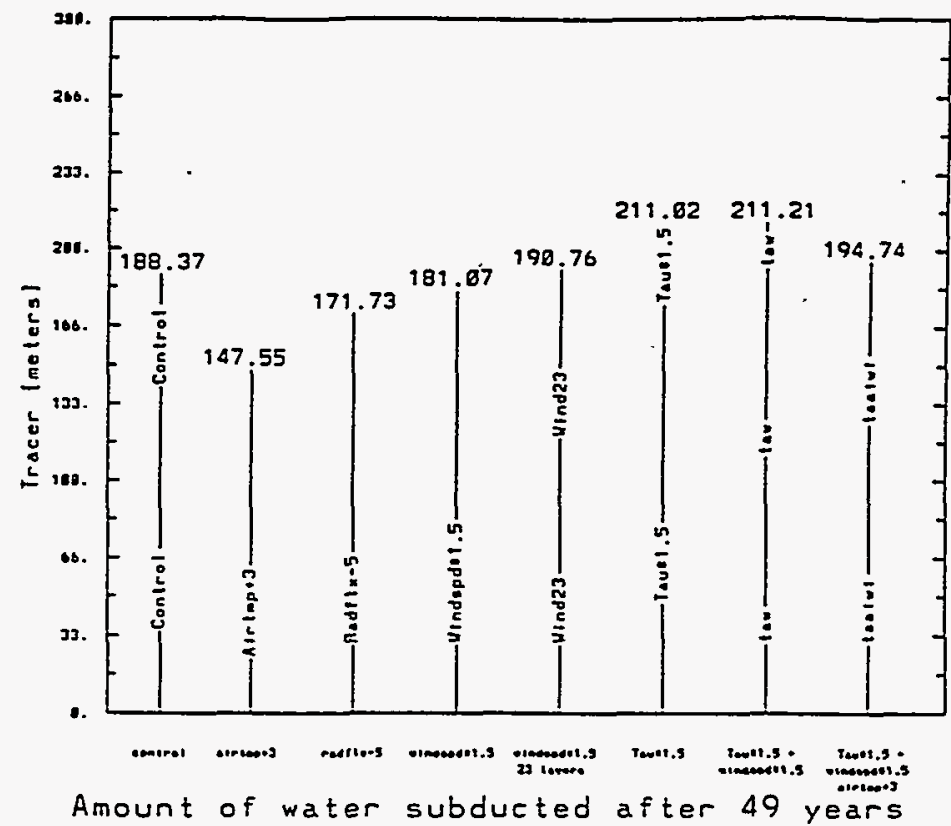

Figure 4.16 Summary of subduction for sector-model experiments..

\section{b. Atlantic-basin model experiments}

As discussed in Section 2 above, the MICOM has undergone almost continuous development since its introduction in the 1989 Bleck et al. paper. The results discussed in the previous section were obtained using a very simplified model appropriate for the North Atlantic basin only because of its latitude range, its initial density structure, and its surface forcing. In order to explore further the effects of varying surface forcing on subduction processes in the North Atlantic, Ms. Horsfall used the version of the MICOM discussed in Bleck et al. (1992). This includes realistic topography and coastlines and a free-surface upper boundary condition (as opposed to the rigid lid in the sector model); in addition, some experiments using diapycnal mixing (i.e., vertical diffusion in the isopycnic framework) were conducted. Further, in contrast to the sector-model experiments, the changes in forcing in the basin-model experiments were dependent on latitude, as shown in Fig. 4.17. In addition, the combined changes in wind and temperature were set to conform more realistically to GCM results pertaining to global-warming 

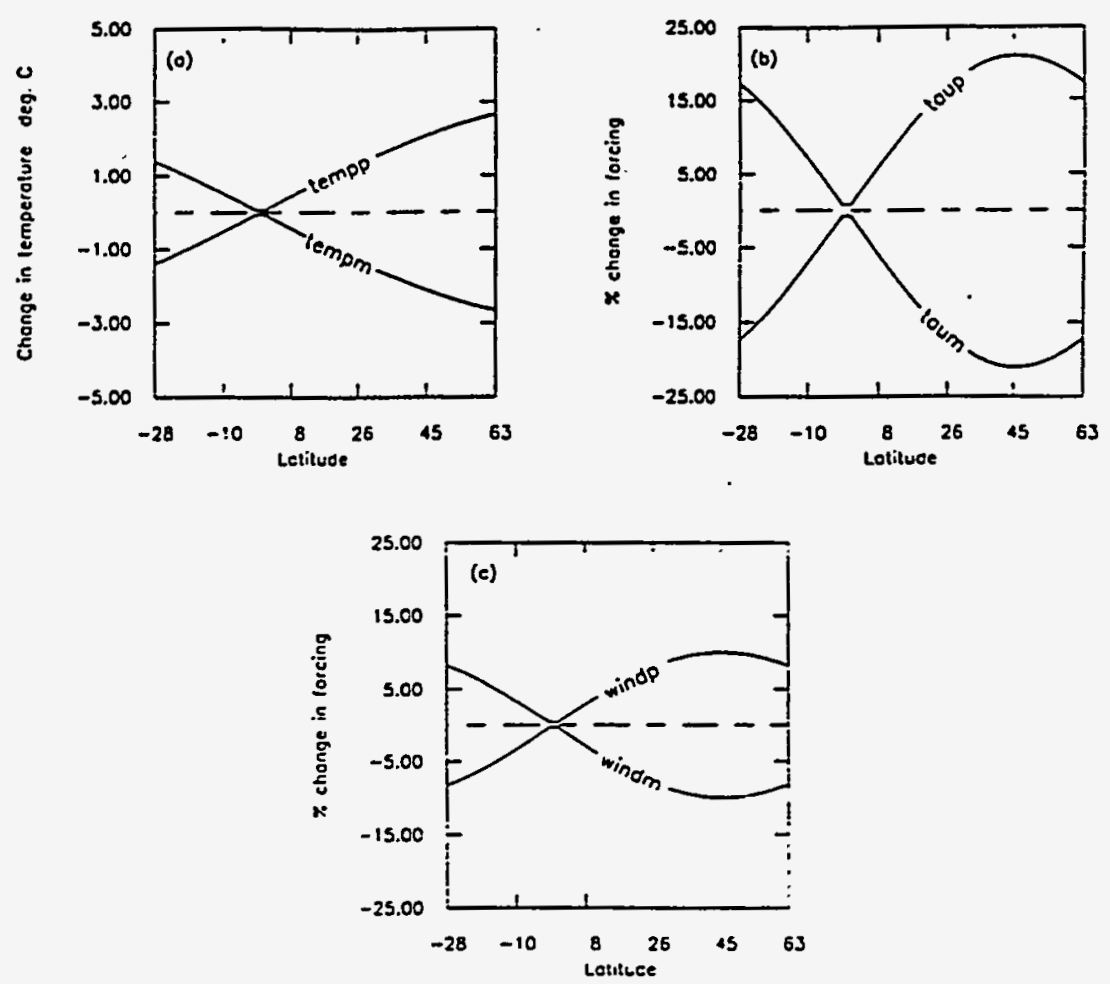

Figure 4.17 Basin-model forcing perturbations as function of latitude for the "plus" ("tempp", "taup", "windp") and "minus" ("tempm", "taum", "windm") experiments; "mixed" is combination of "tempp" with "taum" \& "windm". Curves are symmetric about equator.

scenarios.

Three long-term integrations of the basin model were used to compare with a control integration. The "plus" experiment (meant to simulate global warming) used increased air temperatures and increased winds and wind stresses, as shown in Fig. 4.17. The "minus" experiment was the mirror image of this, using decreased temperatures, winds, and wind stresses. Finally, a "mixed" experiment explored the case of increased air temperatures and decreased 
USDOE Grant No. DE-FG03-93ER61646

Final Report

Page 46
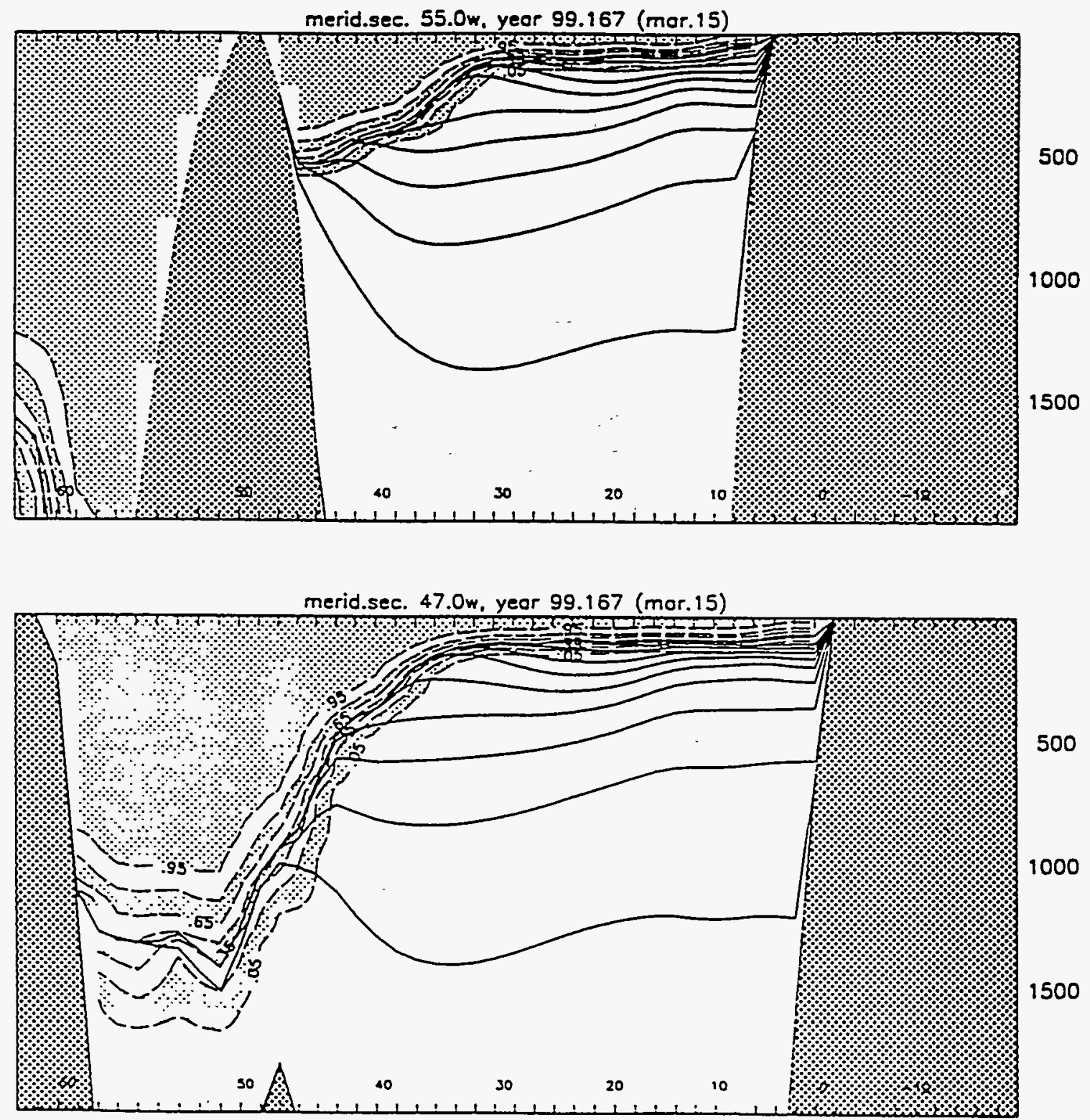

Figure 4.18 Meridional cross-sections in March at $55^{\circ} \mathrm{W}$ (top) and $47^{\circ} \mathrm{W}$ (bottom) for control experiment using basin model. Model layers and $15 \%$ isopleths of tracer are shown. 
USDOE Grant No. DE-FG03-93ER61646

Final Report

Page 47
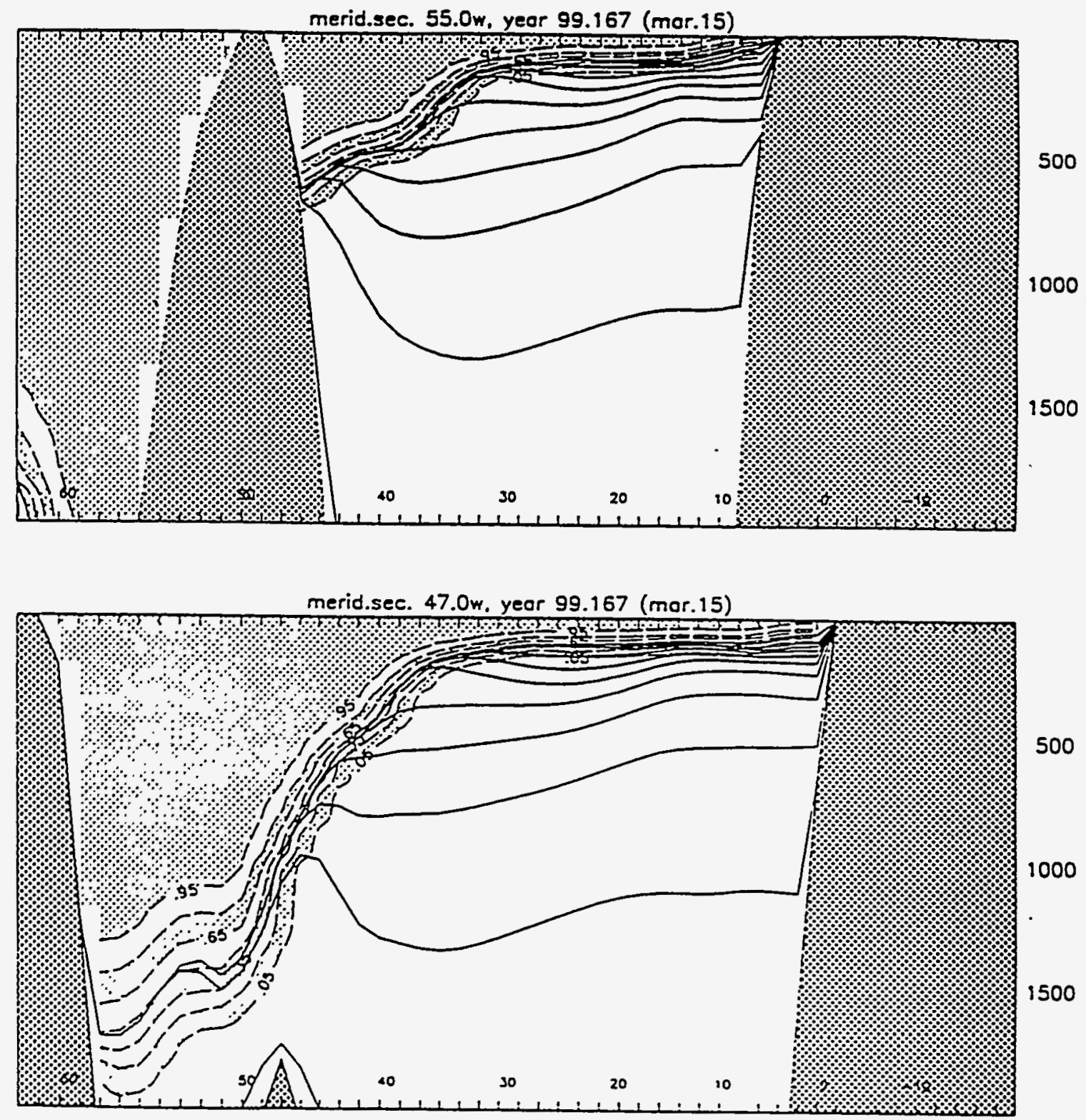

Figure 4.19 Meridional cross-sections in March at $55^{\circ} \mathrm{W}$ (top) and $47^{\circ} \mathrm{W}$ (bottom) for "plus" experiment using basin model. Model layers and 15\% isopleths of tracer are shown. 
USDOE Grant No. DE-FG03-93ER61646

Final Report

Page 48

winds and wind stresses. Although the "mixed" experiment is not completely physically consistent with GCM results, it provides a useful look at the processes that control subduction in the North Atlantic.

Figure 4.18 shows meridional crosssections at $55^{\circ} \mathrm{W}$ and $47 \mathrm{~W}$ for the last March of the control run; layer interfaces are solid lines and tracer isopleths (in

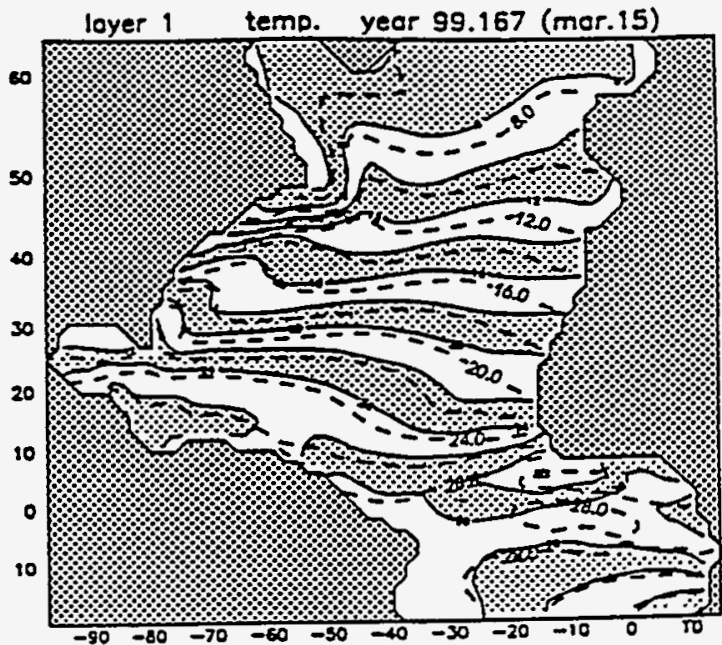
contours of $15 \%$ ) are dashed. The dark stippled area is land, and the light stippled area shows tracer (this is clearer in the lower cross-section). In the $55^{\circ} \mathrm{W}$ crossFigure 4.20 March SSTs for control (dashed) and "plus" experiment (solid). section, the "island" at about $50^{\circ} \mathrm{N}$ is Newfoundland, and in both cross-sections, the northern coast of South America closes the basin (at these longitudes) between the equator and $10^{\circ} \mathrm{N}$. After about 100 years of integration the model has begun to distribute tracer into its interior, via the processes associated with the annual cycle of the mixed layer discussed previously, and the circulation has become nearly steady.

The sector-model results discussed in the section above showed that the combination of increased air temperatures and increased winds effectively canceled, in terms of changes in oceanic subduction associated with the annual cycle. Figure 4.19 verifies this result in the basin model: differences in tracer penetration on the two sections shown between the "plus" experiment and the control are quite small. This is not to suggest that the changes in forcing have little effect on model behavior. The SST changes (Fig. 4.20) reflect increased surface warming, despite greater vertical mixing, and generally higher temperatures particularly in the 
USDOE Grant No. DE-FG03-93ER61646

Final Report

Page 49
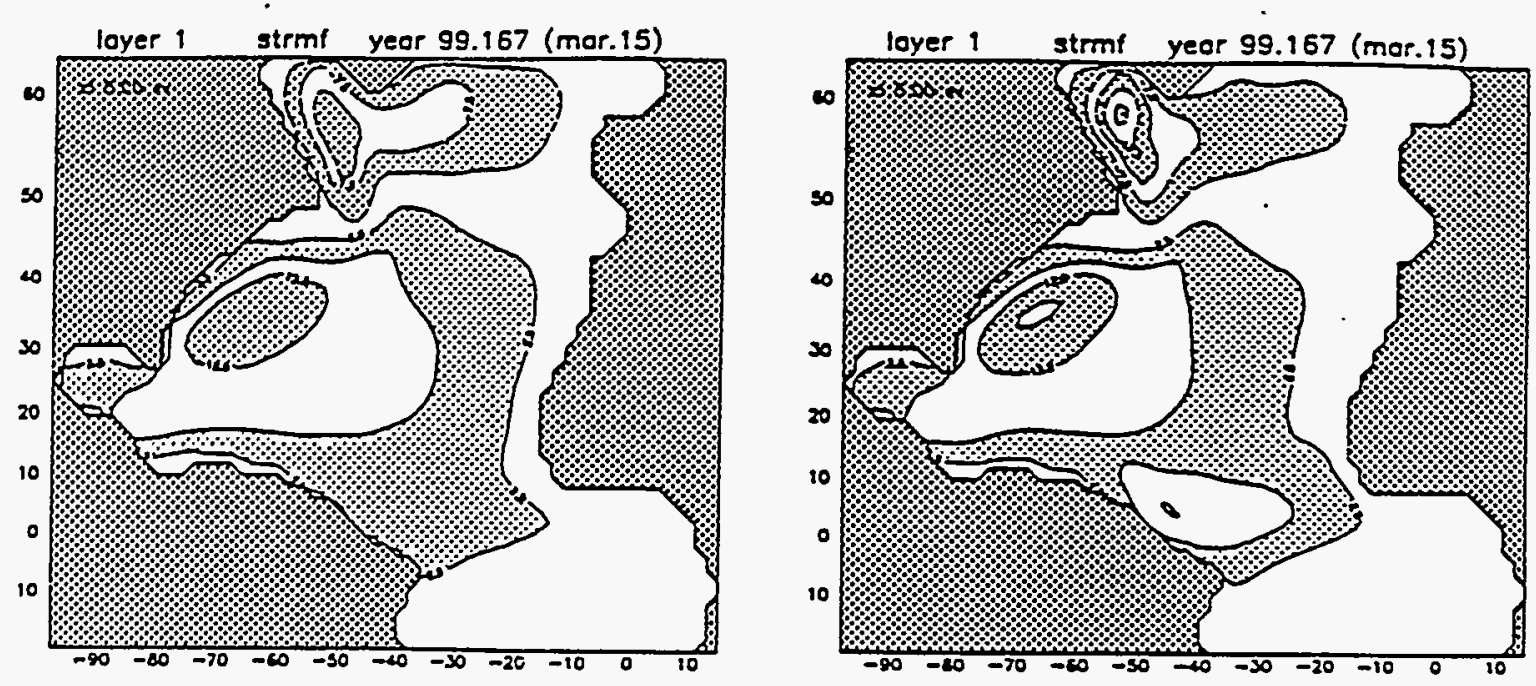

Figure 4.21a Mixed-layer streamfunctions for March for control (left) and "plus" (right) basin model experiments.
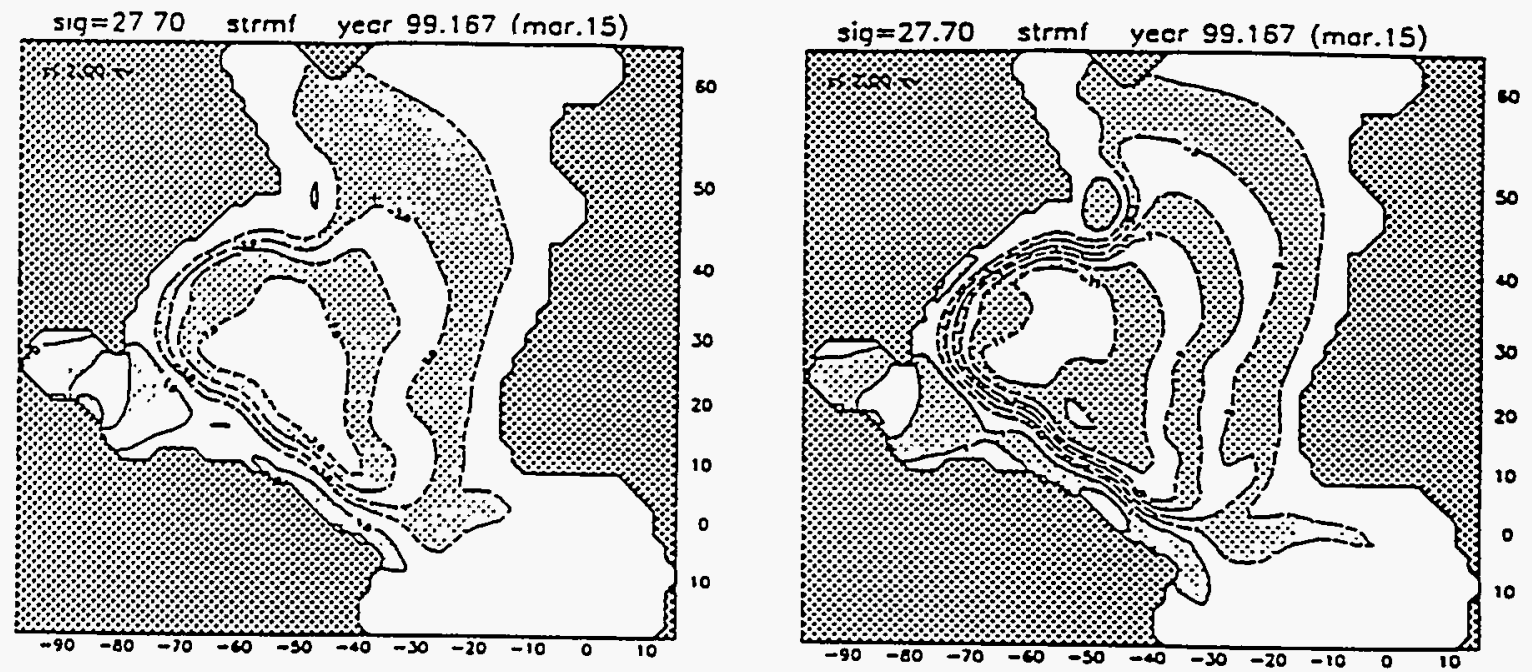

Figure 4.21b Bottom layer streamfunctions for March for control (left) and "plus" (right) basin model experiments. 
USDOE Grant No. DE-FG03-93ER61646

Final Report

Page 50

northern part of the basin. And both the mixed-layer and deep-water stream functions (Figs. 4.21) are more energetic in the "plus" case, consistent with the greater input of wind energy to the circulation.

Interestingly, the cancellation of the (presumed) changes in forcing associated with global warming are not symmetric, as results of the "minus" experiment show. The meridional crosssections (Fig. 4.22) show that increasing surface cooling (via lower air temperatures) with less wind energy input to the model actually results in less mixed-layer penetration and tracer transport into the mid- and deep levels of the model. As expected the circulation is weaker at all levels (Fig. 4.23) as well. This suggests that wind forcing effects exert greater control over deep water production than thermodynamic effects, at least for the magnitudes of the changes used here.

Finally, the "mixed" experiment shows how increased buoyancy input at the surface, which stabilizes the water column, and lower wind energy input can work together to cut off lower levels of the ocean from the effect of surface processes. The cross-sections (Fig. 4.24) show significantly less tracer at the deeper levels, and this can be attributed directly to the shallower wintertime mixed layers in this case. Results of the three experiments with the basin for the deepest model layer are summarized in Fig. 4.25.

This discussion summarizes only part of Horsfall's (1996) project, and aspects of the model sensitivity associated with including additional processes, such as diapycnal mixing, investigated therein are not included in the discussion here. The fundamental results shown above are not altered by these changes, however. 
USDOE Grant No. DE-FG03-93ER61646

Final Report

Page 51
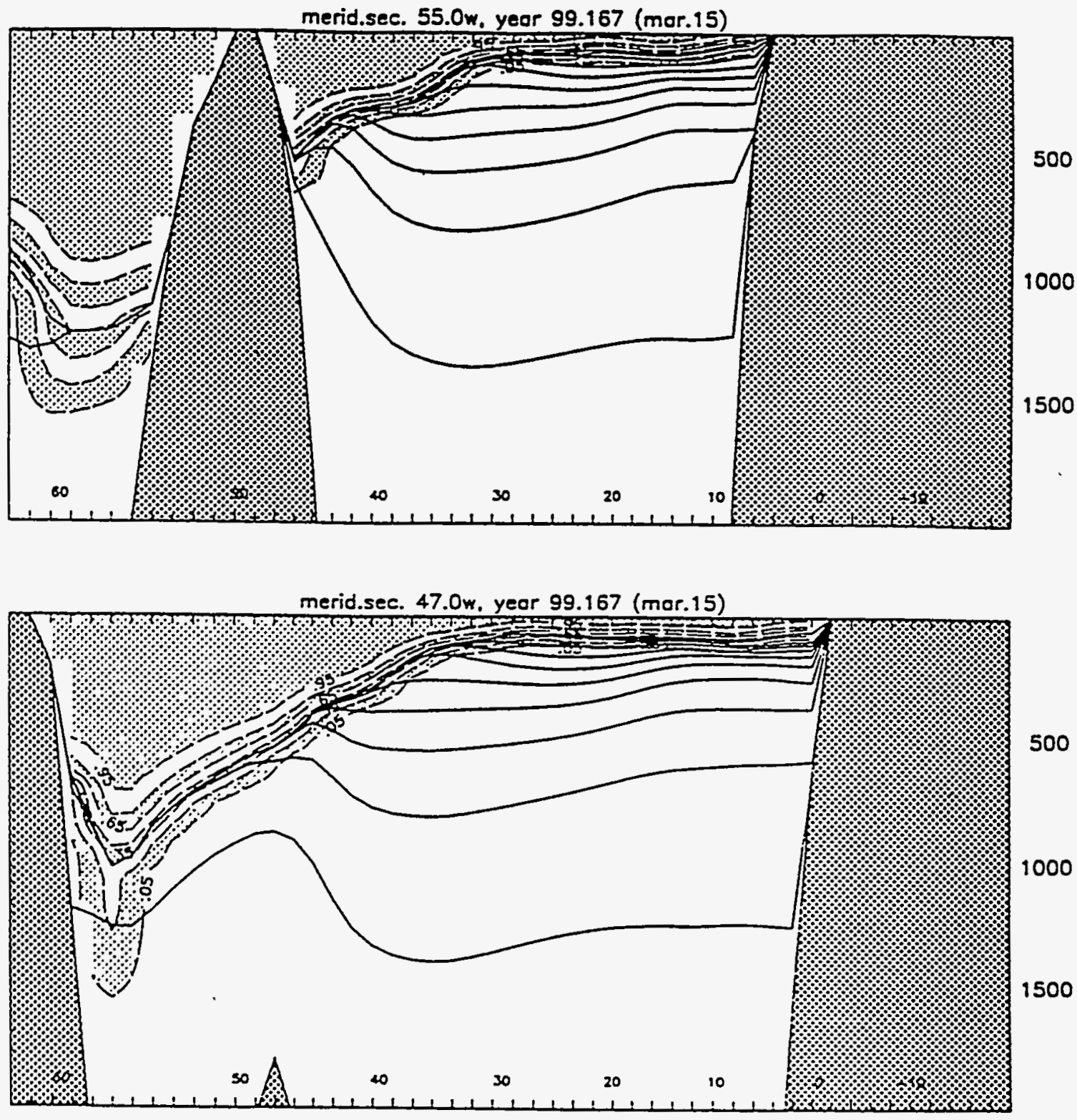

Figure 4.22 Meridional cross-sections in March at $55^{\circ} \mathrm{W}$ (top) and $47^{\circ} \mathrm{W}$ (bottom) for "minus" experiment using basin model. Model layers and $15 \%$ isopleths of tracer are shown. 
USDOE Grant No. DE-FG03-93ER61646

Final Report

Page 52
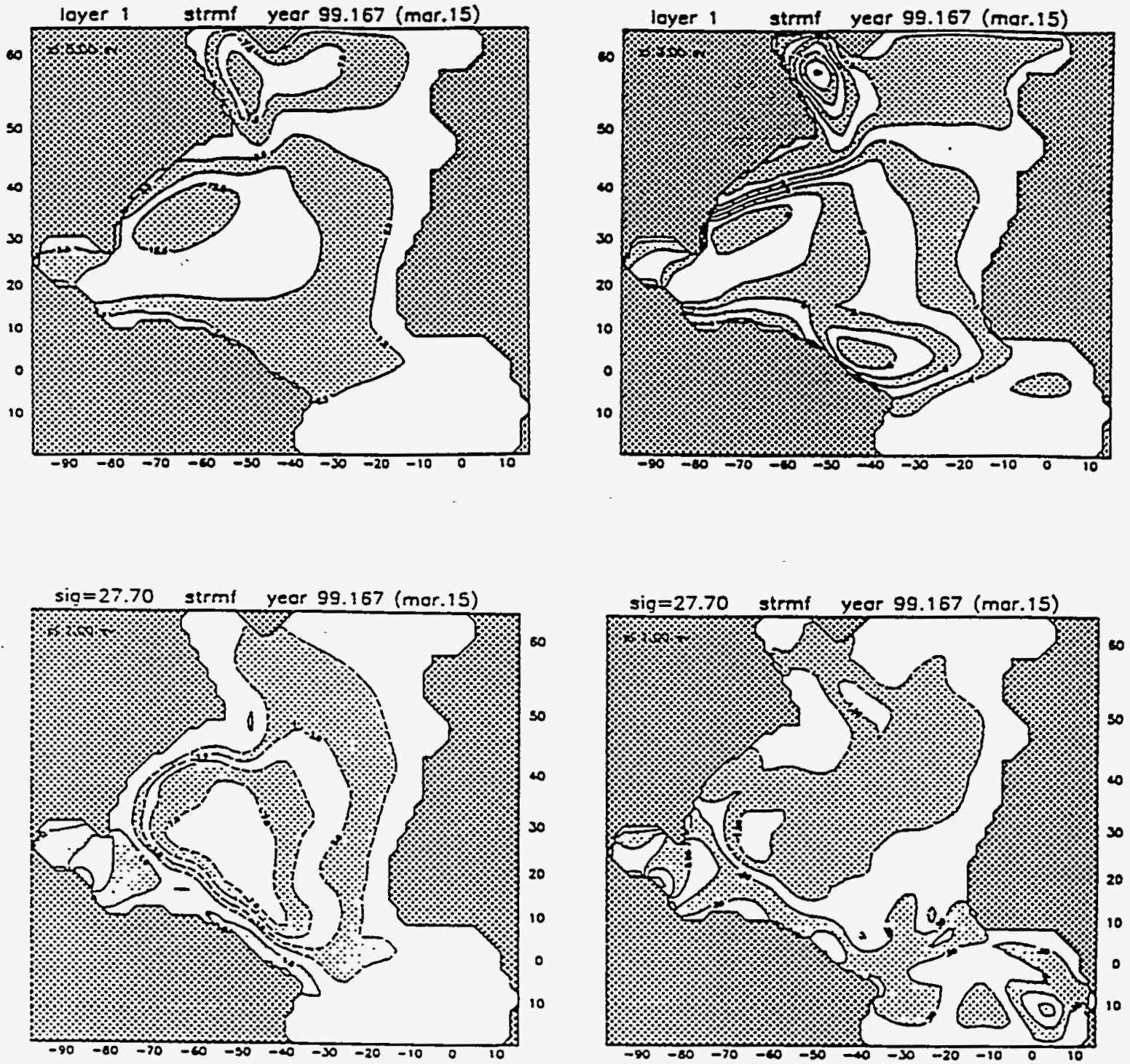

Figure 23 Streamfunctions in mixed layer (top) bottom layer (bottom) for control (left) and "minus" (right) basin model experiments. 
USDOE Grant No. DE-FG03-93ER61646

Final Report

Page 53
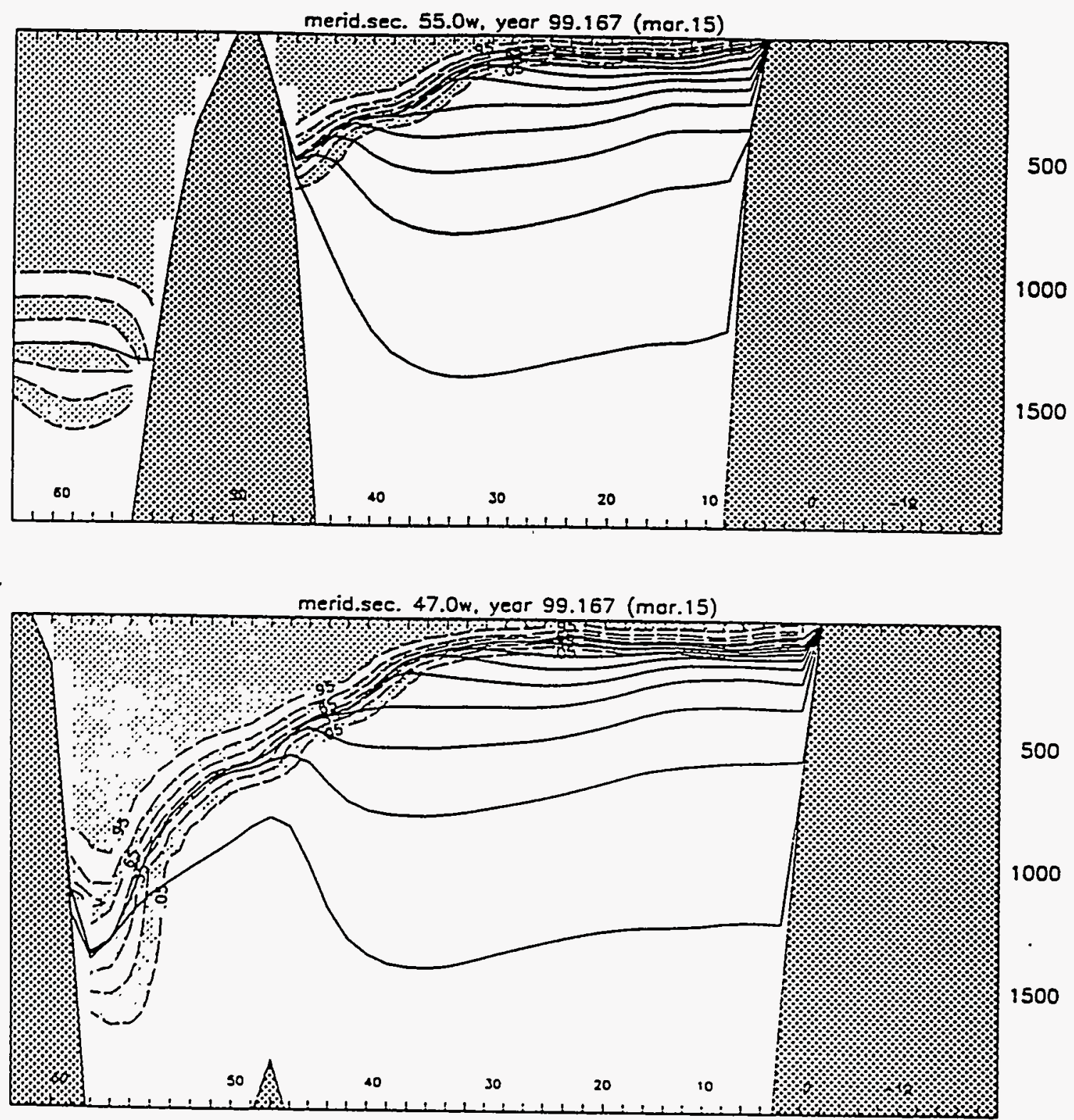

Figure 4.24 Meridional cross-sections in March at $55^{\circ} \mathrm{W}$ (top) and $47^{\circ} \mathrm{W}$ (bottom) for "mixed" experiment using basin model. Model layers and $15 \%$ isopleths of tracer are shown. 

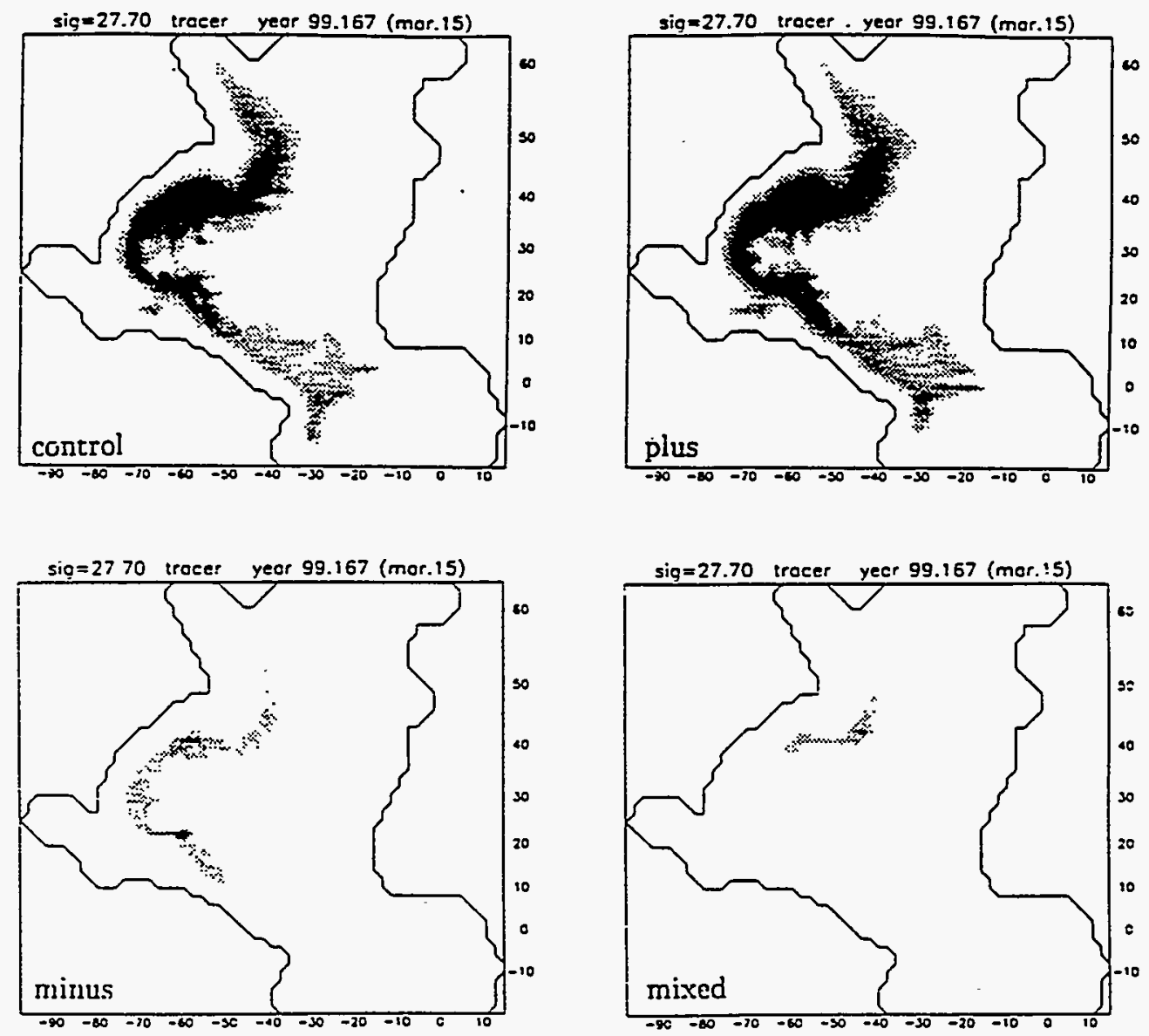

Figure 4.25 Tracer distribution in bottom model layer for various experiments with basin model.

Therefore, it appears that the effects of changes in atmospheric forcing of the oceanic thermohaline circulation associated with global warming will depend crucially on how the surface wind fields respond to the temperature changes. If the winds generally increase, as suggested by some climate model experiments, the oceanic changes could be minimal. However, if they decrease while the temperatures increase, the result would be less sequestration of both heat and chemicals in the seasonal thermocline and the deep ocean. This scenario would imply a positive feedback as originally hypothesized. The magnitude of the feedback cannot by quantified without a physically realistic, coupled global model. 
USDOE Grant No. DE-FG03-93ER61646

Final Report

Page 55

\section{Conclusion}

This project involved using the Miami Isopycnic Coordinate Ocean Model (MICOM) to study the sensitivity of storage of heat and chemicals in the seasonal thermocline to atmospheric forcing of the oceanic circulation. The MICOM has undergone considerable evolution over the duration of the project, and some of this development was supported in part by this award. This support has, with other sources, produced a new and highly flexible tool for the oceanographic and climate research communities

In addition, several conclusions emerge from this work:

- Neglecting sub-monthly correlations of state variables when formulating mean monthly forcing for ocean models can lead to significant errors in forcing.

- It is necessary to compute the saturation mixing ratio of the SST, regardless of computational penalty, to formulate correctly the surface forcing.

- In model calculations using only an oceanic component, surface forcing is best formulated as a mixture of flux and relaxation boundary conditions to simulate as closely as possible the actual behavior of air-sea interaction processes.

- Correct simulation of the annual cycle of the mixed layer requires the inclusion of a diurnal solar cycle as well as wind variability down to at least synoptic scales.

- Thermodynamic considerations alone show that global warming will lead to decreased subduction into the seasonal thermocline.

- Changes in the winds associated with global warming, however, may ameliorate this and, if winds weaken as the climate warms, will add to the decreased subduction.

- Calculations using a fully coupled, global ocean-atmosphere model will be necessary to resolve this issue. 
USDOE Grant No. DE-FG03-93ER61646

Final Report

Page 56

\section{Bibliography}

(* Indicates contributions directly sponsored by USDOE Grant No. DE-FG03-93ER61646)

Berliand, M.E., and Berliand, T.G., 1952: Determining the Net Long-wave Radiation of the Earth with Consideration of the Effect of Cloudiness. Isv. Akad. Nauk. SSSR Ser. Geophys. No. 1.

Bleck, R. 1995: Fine-Scale Modeling of the North Atlantic Ocean. US WOCE Report, 1995, p. 46.

Bleck, R., and D.B. Boudra, 1981: Initial Testing of a Numerical Ocean Circulation Model Using a Hybrid (Quasi-isopycnic) Vertical Coordinate. J. Phys. Oceanogr., 11, 755-770.

Bleck, R., and D.B. Boudra, 1986: Wind-driven Spin-up in Eddy-resolving Models Formulated in Isopycnic and Isoparic Coordinates. J. Geophys. Res. (C), 91, 7611-7622.

Bleck, R., H.P. Hanson, D. Hu and E.B. Kraus, 1989. Mixed Layer-thermocline Interaction in a Three-dimensional Isopycnic Coordinate Model. J. Phys. Oceanogr., 19,1417-1439.

Bleck, R. and L. Smith, 1990: a Wind-driven Isopycnic Coordinate Model of the North and Equatorial Atlantic Ocean. 1. Model Development and Supporting Experiments. $J$. Geophys. Res., 95C, 3273-3285.

Bleck, R., C. Rooth, D. Hu and L.T. Smith, 1992 : Ventilation Patterns and Mode Water Formation in a Wind- and Thermohaline- Driven Isopycnic Coordinate Model of the North Atlantic. J. Phys. Oceanogr., 22, 1486-1505.

Bleck, R., S. Dean, M. O'Keefe and A. Sawdey, 1995: A Comparison of Data-Parallel and Message-Passing Versions of the Miami Isopycnic Coordinate Ocean Model (MICOM). Parallel Computing, 21, 1695-1720.

Bleck, R., and S. Dean, 1995: Ocean Modeling Using Isopycnic Coordinates. LALP-95-239, Los Alamos National Laboratory.

Bleck, R., and A. New, 1995: an Isopycnic Model Study of the North Atlantic. Part II: 
USDOE Grant No. DE-FG03-93ER61646

Final Report

Page 57

Interdecadal Variability of the Subtropical Gyre. J. Phys. Oceanogr., 25; 2700-2714.

Bleck, R., S. Sun, and S. Dean, in press: Global Ocean Simulations with an Isopycnic Coordinate Model. In: Some New Directions in Science on Computers. World Scientific Press, Singapore.

Bleck, R. 1996: MICOM-Based Global Modelling in the US. International WOCE Newsletter, No. 23, 31-33.

Budyko, M.I., 1974: Climate and Life. Academic Press, New York, 508pp. :

Gaspar, P., 1988: Modeling the Seasonal Cycle of the Upper Ocean. J. Phys. Oceanogr., 18,161-180.

Goff, J.A., and S. Gratch, 1946: Low-pressure properties of water from -160 to $212^{\circ} \mathrm{F}$. Trans. ASHVE, 52, 95-121.

*Halliwell, G.R., H.P. Hanson, F. Horsfall, and R. Bleck 1994: Sensitivity of an IsopycnicCoordinate OGCM to Parameterizations of Upper-Ocean Mixing. EOS: Transactions of the American Geophysical Union, 75, (3, Suppl.), p. 189.

Haney, R., 1971: Surface thermal boundary condition for ocean circulation models. J. Phys. Oceanogr., 1, 241-248.

*Hanson, H.P, 1994: Modeling Climatological Heat Storage in the North Atlantic Ocean. Eos: Transactions of the American Geophysical Union, 75, (3, Suppl.), pp. 152-153.

*Hanson, H.P., 1995a: Fuzzy Cloud Concepts for Assessing Radiation Feedbacks. In Climate -Radiation Feedbacks: The Current State of the Science. DOE/ER-0661T, U.S. Dept. of Energy, Washington DC, pp. 44-47.

*Hanson, H.P., 1995b: Natural Boundary Conditions for Ocean Climate Modeling. Abstracts, International Union of Geodesy and Geophysics XXI General Assembly, A328 [Abstract of poster for the 1995 IUGG General Assembly]

*Hanson, H.P., 1995cNatural Boundary Conditions for the Global MICOM. Abstracts, Second MICOM Workshop, February 1995, Miami, FL [Available from the Rosenstiel School for Marine and Atmospheric Science, University of Miami]. 
USDOE Grant No. DE-FG03-93ER61646

Final Report

Page 58

*Hanson, H.P., C. Penland, and R. Bleck, 1996:Vertical Mixing Controls on Seasonal Thermocline Formation. Eos: Transactions of the American Geophysical Union, 76, 3 (Suppl.) p. OS151.

*Hanson, H.P., and R. Bleck, 1996: The Well-Seasoned MICOM: Forcing the Annual Cycle of SST.

*Horsfall, F., R. Bleck, and H.P. Hanson, 1994a: Variation of the Subduction Rate in Response to Global Climate Change. Eos: Transactions of the American Geophysical Union, 75, (3, Suppl.), p. 161.

*Horsfall, F., R. Bleck, and H.P. Hanson, 1994b: Variation of the Subduction Rate in Response to Global Climate Change Abstracts, First MICOM Workshop, February, 1994 [Available form the Rosenstiel School of Marinde and Atmospheric Science, University of Miami]

*Horsfall, F., 1996: The effect of variable atmospheric forcing on oceanic subdction of a passive tracer in a numerical model: Implications for global warming. Ph.D. dissertation, Rosenstiel School of Marine and Atmospheric Sciences, University of Miami, Miami, FL.

*Horsfall, F., R. Bleck, and H.P. Hanson, 1997: The Effect of Variable Atmospheric Forcing on Oceanic Subduction of a Passive Tracer in a Numerical Model: Implications for Global Warming. Extended Abstracts, Seventh Conference on Climate Variations of the American Meteorological Society, Long Beach CA February 2-7, 1997. [In press]

$\mathrm{Hu}$, Dingming, 1991: Diapycnal mixing in a joint mixed-layer/isopycnic coordinate numerical model of the wind- and thermohaline-driven ocean general circulation. Ph.D Dissertation, Rosenstiel School of Marine and Atmospheric Science, University of Miami, Miami, FL.

Kleeman, R., and S.B. Power, 1995: A simple atmospheric model of surface heat flux for use in ocean modeling studies. J. Phys. Oceanogr., 25, 92-105.

Kraus, E.B. and J.S. Turner, 1967: A one-dimensional model of the seasonal thermocline. Part 
USDOE Grant No. DE-FG03-93ER61646

Final Report

Page 59

II: The general theory and its consequences. Tellus, 19, 98-106.

Kraus, E.B., R. Bleck, and H.P. Hanson, 1988: The Inclusion of a Surface Mixed Layer in a Large-Scale Circulation Model. In: Small Scale Turbulence and Mixing in the Ocean, J.C.J. Nihoul and B.M. Jamart, Eds., Elsevier Science Publishers, New York, pp. 51-62.

Lowe, P.R., 1977: An approximating polynomial for the computation of saturation vapor pressure. J. Appl. Meteorol., 16, 100-103.

Manabe, S., and R.T. Wetherald, 1975: The effects of doubling the $\mathrm{CO}_{2}$ concentration on the climte of a general circulation model. J. Atmos. Sci., 32, 3-15.

Manabe, S. and R.J. Stouffer, 1994: Multiple-century response of a coupled ocean-atmosphere model to an increase in atmospheric carbon dioxide. J. Clim., 7, 5-23.

Manabe, S., R.J. Stouffer, M.J. Spelman and K. Bryan, 1991: Transient reponses of a coupled ocean-atmosphere model to gradual changes of atmospheric $\mathrm{CO}_{2}$. Part I: Annual mean response. J. Clim., 4, 785-818.

Manabe, S., R.J. Stouffer and M.J. Spelman, 1992: Transient repsonses of a coupled oceanatmosphere model to gradual changes of atmospheric $\mathrm{CO}_{2}$. Part II: Seasonal response. J. Clim., 5,105-126.

Niiler, P.P., and E.B. Kraus, 1977: One-dimensional models of the upper ocean. In: Modelling and Prediction of the Upper Layers of the Ocean. Ed. E.B. Kraus, Pergamon Press, New York. pp. 143-172.

Oberhuber, J.M., 1988: An Atlas Based on the 'COADS' Data Set: The Budgets of Heat, Buoyancy and Turbulent Kinetic Energy at the Surface of the Global Ocean. Max-PlanckInstitut für Meteorologie Report No. 15, Hamburg, Germany.

Oberhuber, J.M., 1993: Simulation of the Atlantic circulation with a couple sea ice - mixed layer - isopycnal general circulation model. Part I: Model description. J. Phys. Oceanogr., 23, 808-829.

Rooth, C., and L. Xie, 1992: Air-sea boundary layer dynamics in the presence of meso-scale surface currents. J. Geophys. Res., 97, 14 431-14 438. 
USDOE Grant No. DE-FG03-93ER61646

Final Report

Page 60

Semtner, A., and R. Chervin, 1992: Ocean general circulation from a global eddy-resolving model. J. Geophys. Res., 97, 5493-5550.

Smith, S.A., 1988: Coefficients for sea surface wind stress, heat flux, and wind profiles as a function of wind speed and temperature. J. Geophys. Res., 93, 15467-15472.

Trenberth, K.E., W.G. Large, and J.G. Olson, 1989: The effective drag coefficient for evaluating wind stress over the oceans. J. Climate, 2, 1507-1516.

Woodruff, S.D., R.J. Slutz, R.L. Jenne, and P.M. Steurer, 1987: A comprehensive oceanatmosphere data set. Bull. Amer. Meteor. Soc., 68, 1239-1250. 